ARTICLE

DOI: $10.1038 / \mathrm{s} 41467-017-01845-1$

\title{
Notch transactivates Rheb to maintain the multipotency of TSC-null cells
}

Jun-Hung Cho , Bhaumik Patel ${ }^{1}$, Santosh Bonala1, Sasikanth Manne ${ }^{1}$, Yan Zhou ${ }^{2}$, Surya K. Vadrevu', Jalpa Patel ${ }^{1}$, Marco Peronaci', Shanawaz Ghouse ${ }^{1}$, Elizabeth P. Henske ${ }^{3}$, Fabrice Roegiers ${ }^{4}$, Krinio Giannikou ${ }^{5}$, David J. Kwiatkowski ${ }^{5}$, Hossein Mansouri ${ }^{6}$, Maciej M. Markiewski ${ }^{1}$, Brandon White $^{7} \&$ Magdalena Karbowniczek (1) ${ }^{1}$

Differentiation abnormalities are a hallmark of tuberous sclerosis complex (TSC) manifestations; however, the genesis of these abnormalities remains unclear. Here we report on mechanisms controlling the multi-lineage, early neuronal progenitor and neural stem-like cell characteristics of lymphangioleiomyomatosis (LAM) and angiomyolipoma cells. These mechanisms include the activation of a previously unreported Rheb-Notch-Rheb regulatory loop, in which the cyclic binding of Notch1 to the Notch-responsive elements (NREs) on the Rheb promoter is a key event. This binding induces the transactivation of Rheb. The identified NRE2 and NRE3 on the Rheb promoter are important to Notch-dependent promoter activity. Notch cooperates with Rheb to block cell differentiation via similar mechanisms in mouse models of TSC. Cell-specific loss of Tsc1 within nestin-expressing cells in adult mice leads to the formation of kidney cysts, renal intraepithelial neoplasia, and invasive papillary renal carcinoma.

\footnotetext{
${ }^{1}$ Department of Immunotherapeutics and Biotechnology, School of Pharmacy, Texas Tech University Health Science Center, 1718 Pine Street, Abilene, TX 79601, USA. ${ }^{2}$ Bioinformatics and Biostatistics Facility, Fox Chase Cancer Center, 333 Cottman Avenue, Philadelphia, PA 19111, USA. ${ }^{3}$ Division of Pulmonary and Critical Care Medicine, Brigham and Women's Hospital and Harvard Medical School, 45 Francis Street, Thorn Building, Room 826, Boston, MA 02115, USA. ${ }^{4}$ Fox Chase Cancer Center, 333 Cottman Ave, Philadelphia, PA 19111, USA. ${ }^{5}$ Division of Pulmonary and Critical Care Medicine, Brigham and Women's Hospital, Harvard Medical School and Dana Farber Cancer Institute, 20 Shattuck Street, Thorn Building 826C, Boston, MA 02115, USA. ${ }^{6}$ Department of Mathematics and Statistics, Texas Tech University, 1108 Memorial Circle, Lubbock, TX 79409, USA. ${ }^{7}$ Department of Biological Sciences, San Jose State University, One Washington Square, San Jose, CA 95192, USA. Correspondence and requests for materials should be addressed to B.W. (email: brandon.white@sjsu.edu) or to M.K. (email: magdalena.karbowniczek@ttuhsc.edu)
} 
T he heterogeneity of cancers reflects the aberrant cell differentiation ${ }^{1,2}$. Poor differentiation of tumor cells often indicates aggressive behavior and stem cell-like properties ${ }^{3}$. The differentiation abnormalities are a hallmark of the central nervous system and peripheral lesions of the tuberous sclerosis complex (TSC), which is a genetic disorder resulting from the loss of TSC1/2 function, manifesting in the form of brain tumors with aberrant glioneuronal differentiation, pulmonary lymphangioleiomyomatosis (LAM), and renal angiomyolipomas ${ }^{4}$. The differentiation plasticity of TSC tumor cells is supported by the expression of melanocytic and smooth muscle markers ${ }^{5}$ and the common origin of vascular, smooth muscle, and fat components of angiomyolipoma ${ }^{6}$. However, the mechanisms behind this plasticity are unclear. Since melanocytes and some smooth muscle cells derive from the neural crest (NC) and LAM and angiomyolipoma express melanocytic and smooth muscle markers, we postulate that the mechanisms regulating NC differentiation might also operate in LAM and angiomyolipoma. The Notch signaling pathway regulates $\mathrm{NC}$ cell differentiation, maintains neural precursors in an undifferentiated state, and impacts cell proliferation and migration during normal development and in cancer $^{7-16}$. The involvement of Notch in TSC pathogenesis has been suggested by studies demonstrating that Rheb activates Notch in angiomyolipoma-derived cells and that TSC proteins regulate the Notch-dependent cell fate decisions during Drosophila sensory organ development ${ }^{17}, 18$. The oscillation in Notch signaling maintains neuronal progenitors in undifferentiated state ${ }^{19}$. Our data imply that angiomyolipoma cells do not achieve terminal differentiation and remain as neural stem-like cells or progenitors; therefore, we explore the possibility of oscillatory Notch1 signaling gene expression as an underlying mechanism blocking angiomyolipoma cell differentiation.

Here we describe a novel Rheb-Notch-Rheb loop and its role in abnormal differentiation of LAM and angiomyolipoma cells that resemble neural stem cells (NSCs) and neuronal progenitors. The elements of this loop include Rheb, which activates Notch 17,18 , and the previously unreported direct binding of Notch 1 to the Rheb promoter. We identified four potential recombination signal binding proteins for immunoglobulin kappa J region (RBPJ) binding sites within the promoter of Rheb. We discovered that binding of Notch1 to the two Notch1-responsive elements (NREs), NRE2 and NRE3, regulates the transcription of Rheb in a cyclic manner and is essential for Notch-dependent expression of Rheb, indicating that Notch1 is a direct and upstream regulator of Rheb, in addition to the tuberin GTPase-activating protein (GAP) domain $^{20}$. The dysregulation of this mechanism leads to the retention of the NSC-like potential of angiomyolipoma cells and TSC tumorigenesis.

\section{Results}

Neural crest markers in LAM and angiomyolipoma. Clinical evidence and the expression of melanocytic and smooth muscle markers point to LAM and angiomyolipoma differentiation plasticity along $\mathrm{NC}$ lineages $5,6,21$. Other cell types in addition to melanocytes and smooth muscle cells, including neurons and glial cells of the peripheral nervous system, originate from the $\mathrm{NC}^{10}$. Therefore, we determined whether the LAM and angiomyolipoma differentiation plasticity involves other NC lineages.

Neuron-specific enolase (NSE) and glial fibrillary acidic protein (GFAP) were expressed in TSC-associated and sporadic angiomyolipoma and LAM, but not in normal adjacent tissue (Fig. 1a-c). Although NSE is not exclusively a neuronal marker, it identifies cells of neuronal and neuroendocrine origin. The expression of neuron-specific tubulin (NS-tubulin) within small clusters of angiomyolipoma supports the neuronal or melanocyte nature of these cells (Fig. 1b and Supplementary Fig. 1A) ${ }^{22}$. In addition to angiomyolipoma, the expression of NS-tubulin was present in papillary micro adenoma from the same patient (Supplementary Fig. 1A, fourth panel). In the normal kidney NStubulin staining was detected only in peripheral nerves, as it should be, confirming high specificity of this assay (Supplementary Fig. 1A, first panel). Nestin, a typical NSC marker ${ }^{23}$, also detected in various cancer cells of neuronal and non-neuronal origin $^{24}$, was expressed in small angiomyolipoma clusters (Fig. 1b), and LAM cells (Fig. 1d). The expression of GFAP, NSE, and nestin in available angiomyolipoma tumors and lack of or very low expression in corresponding normal kidneys was confirmed by western immunoblotting (Fig.1c(i, ii)), Supplementary Fig. 1E-ii and Supplementary Fig. 10). Nestin and the neuronal marker peripherin ${ }^{25}$ were co-expressed in LAM and angiomyolipoma, but not in normal adjacent cells (Fig. 1d, Supplementary Fig. 1B and Supplementary Table 1).

The differentiation plasticity of angiomyolipoma is supported by the co-expression of multiple NC markers ${ }^{26}, 27$ in angiomyolipoma-derived cell lines ${ }^{28-30}$ determined by fluorescence-activated cell sorting (FACS) (Fig. 1e and Supplementary Fig. 1C). For comparison, we used NC-derived melanoma cells (Supplementary Fig. 1D), expecting that NC markers will be expressed in these cells. Tuberin (TSC1 product) and hamartin (TSC2 product) expression was examined by western blotting (WB) in angiomyolipoma-derived cells (Supplementary Fig. 1E). The upregulation of mammalian target of rapamycin (mTOR) signaling in angiomyolipoma cells was confirmed by WB (Supplementary Fig. 1E, F, and H), real-time reverse transcriptase-PCR (RT-PCR) (Supplementary Fig. 1G), and global transcriptomic analysis of angiomyolipoma and angiomyolipoma-derived cells vs. the corresponding normal kidney (Supplementary Fig. 2 and Supplementary Table 2).

Through FACS (Fig. 1e(i) and Supplementary Fig. 1C-D), we have identified subpopulations of cells with profiles similar to either the NSC-like cells or multipotent progenitors. These populations consist of: (1) cells expressing nestin alone, similar to $\mathrm{NSC}^{10,31}$; (2) cells co-expressing nestin and melan-A, or NStubulin, indicating melanocyte or neuronal differentiation, respectively; and (3) cells co-expressing nestin, GFAP, and melan-A, reflecting glial or melanocyte differentiation ${ }^{32}$ (Fig. 1e (i) and Supplementary Fig. 1C). The expression of nestin, NStubulin, and GFAP in angiomyolipoma-derived cells was verified by WB (Fig. 1e(ii)). The NSC-like profile of angiomyolipoma cells was inter-related with the expression of transcription factors maintaining the multipotency of NSC and preventing differentiation, such as SoxE and inhibitor of DNA binding (ID) subfamily ${ }^{2}$, 33, SOX10, SOX9, and ID3 (Fig. 1f). Angiomyolipoma cells also have the potential to differentiate along Schwann and melanocytic lineages, since they expressed S100A1 (Schwann), DCT, and c-Kit (melanocytic) markers ${ }^{34,35}$ (Fig. 1f). These data suggest that angiomyolipoma cells with mutated TSC1/2 $28-30$ and/or hyperactivated Rheb ${ }^{28-30}$ (Supplementary Fig. 1E-H) have a similar profile to NSC-like cells and early multipotent progenitors.

To support differentiation plasticity of angiomyolipoma through unbiased approach, we performed a global transcriptomic analysis of sporadic angiomyolipoma, corresponding normal kidney, and two angiomyolipoma-derived cell lines using RNA sequencing (RNA-seq). Three gene lists were intersected, and only common genes which were differentially expressed in angiomyolipoma and angiomyolipoma cells relative to normal kidney (Supplementary Fig. 2) were used for the gene ontology (GO) enrichment analysis. The analysis of the significant genes was performed using GOstats package (Bioconductor) ${ }^{36}$. The enriched biological process were defined by $P$-value cutoff of $<0.001$ using hypergeometric test with GOstats package, and only 
the enriched categories related to neurogenesis, multipotency, and differentiation are shown. These processes include axon extension, regulation of neuron and cell differentiation, morphogenesis, and nervous system development (Supplementary Fig. 3). Using pathway analysis, we found alterations in expression of genes involved in stem cell pluripotency, melanocyte pigmentation, and renal cell carcinoma (RCC) signaling (Supplementary Table 2). In addition, the bio-function categories enriched for differentially expressed genes in angiomyolipoma tumor and angiomyolipoma cell lines are shown (Supplementary Table 3). In conclusion, these analyses point to differentiation plasticity of angiomyolipoma and potential of tumor cells to differentiate into NC-derived tissues and cells.

Since activation of mTOR is the driver of TSC tumorigenesis, we assessed the effect of rapamycin (an mTOR inhibitor) on the NSC-like profile of angiomyolipoma cells. Although rapamycin reduced mTORC1 activation because it decreased the number of cells containing phosphorylated ribosomal protein 6 (pS6), which is mTORC1 downstream target (Supplementary Fig. 5A), the number of nestin-positive NSC-like cells did not change (Supplementary Fig. 5B). Instead, rapamycin shifted the quiescent angiomyolipoma NSC-like cells $\left(\right.$ nestin $^{+} \mathrm{Ki67}^{-}$) from $\mathrm{G}_{\text {alert }}$ a

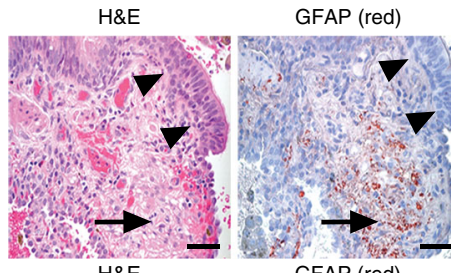
$H \& E$

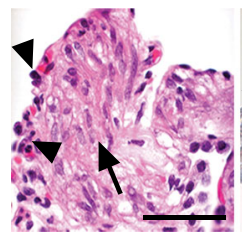

C

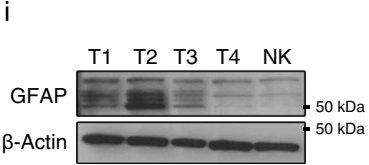

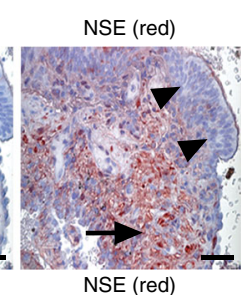

GFAP (red)
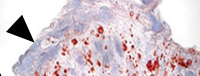

ne.
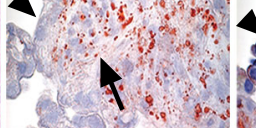

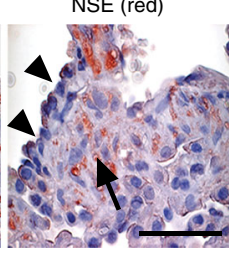

ii b

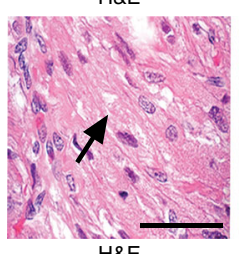

$H \& E$
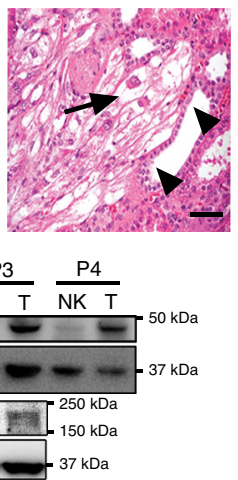
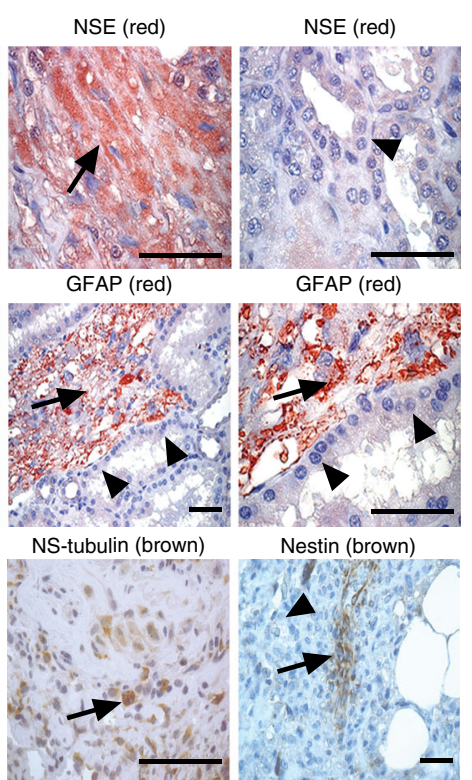

d
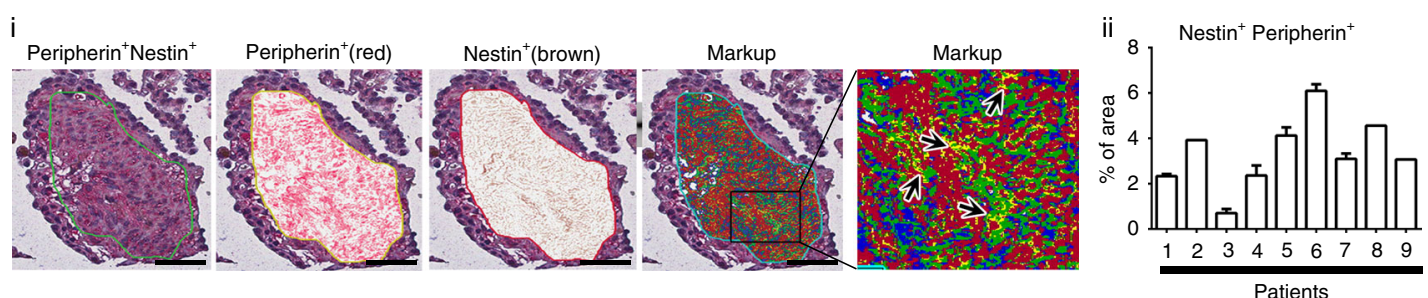

e
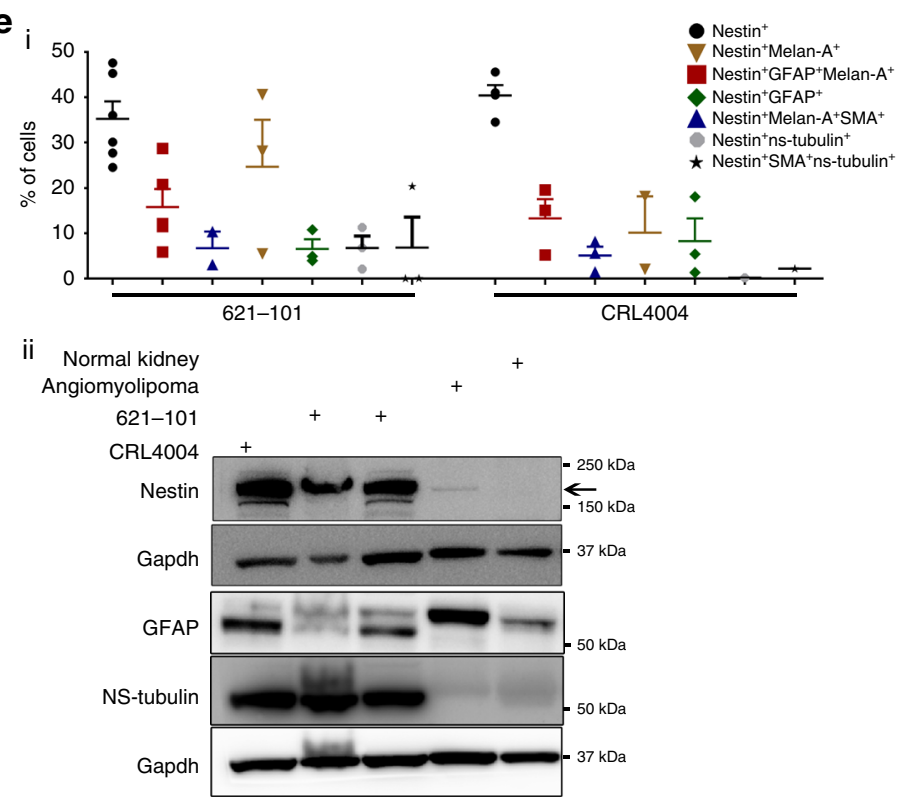

f Angiomyolipoma 621-101 +

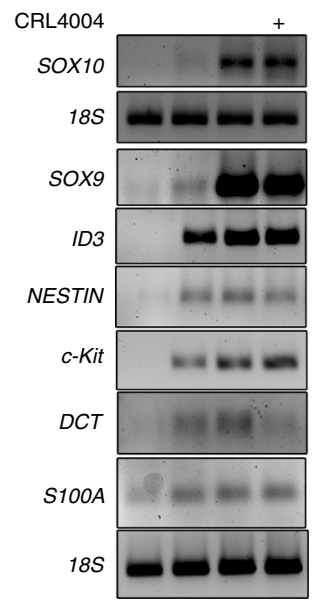


$\left(\right.$ nestin $^{+} \mathrm{Ki}^{-} 7^{-} \mathrm{pS6}^{+}$) to $\mathrm{G}_{0}\left(\right.$ nestin $^{+} \mathrm{Ki67}^{-} \mathrm{pS6}^{-}$) (Supplementary Fig. 5C), consistent with the role of mTORC1 in 'alerting' quiescent stem cells $^{37}$. These data also suggest that aberrant differentiation of angiomyolipoma is not caused by mTOR upregulation.

The oscillation of RHEB and HES1 in angiomyolipoma cells. Oscillatory gene expression regulates cell cycle ${ }^{38}$, somitogenesis ${ }^{39}$, and neural progenitor differentiation ${ }^{19}$. Given the role of Notch signaling oscillation in maintaining the multipotency of NSCs and progenitors ${ }^{19}$ in conjunction with the phenotype of angiomyolipoma cells (Fig. 1 and Supplementary Figs. 1-3), and roles of Rheb and Notch in TSC tumorigenesis ${ }^{17,18}$, we theorized that cycling of Rheb and Notch signaling in NSC-like angiomyolipoma cells prevents their differentiation. We looked at the endogenous RHEB and HES1 messenger RNAs (mRNAs) by quantitative reverse transcriptase-PCR ( $\mathrm{q}(\mathrm{RT})-\mathrm{PCR}$ ) in angiomyolipoma-derived cells, synchronized as previously described ${ }^{40,41}$. RHEB mRNA oscillated, with peaks at 30,120 , and 210, or 60,120, and $240 \mathrm{~min}$, after addition of serum, which initiated oscillation (Fig. 2a(i, iii), Supplementary Fig. 6A, and Supplementary Fig. 8A-i, B-i), with some variability between used cell lines, and reached a maximum at 120 , 210, or $240 \mathrm{~min}$ (Fig. 2a(i, iii) and Supplementary Fig. 6A, 8A-i, Bi). HES1 mRNA oscillated with peak at 90 min (Fig. 2a(ii, iv) Supplementary Fig. 6B, and Supplementary Fig. 8A-ii, B-ii). The observed RHEB and HES1 oscillation was not related to changes in GAPDH (Supplementary Fig. 6C and D, black lines). We corroborated $\mathrm{qPCR}$ data by WB (Fig. 2b(i-ii) and Supplementary Fig. 10). The oscillation of Hes1 depends on Notch signaling ${ }^{19}$. Therefore, similarity in the oscillation patterns (regardless of differences in the magnitude of oscillation) of Rheb and Hes1 led us to hypothesize that Notch1 also regulates the changes in RHEB mRNA expression. We searched for potential RBPJ binding sites within the human Rheb promoter, since these elements bind the Notch1 ternary complex, to regulate the target gene promoters ${ }^{42}$. We identified four potential RBPJ binding sites, with the last site located within the exon 1 (Fig. 2c(i)). We used chromatin immunoprecipitation qPCR (ChIP-qPCR) to examine the binding of Notch1 to the promoter of Rheb and Hes1 (as a positive control) in angiomyolipoma cells in Dulbecco's modified Eagle's medium (DMEM) to identify which sites are functionally important and correspond to true NREs. We also measured the activity of the Rheb promoter using luciferase-reporter gene assays in the presence or absence of the Notch1 ternary complex members: mastermind-like 1 (MAML1) and Notch1 intracellular domain (N1ICD). Notch1 bound mainly to the NRE2 and NRE3 within the Rheb (Fig. 2c(ii, iv)) and Hes1 promoters (Fig. 2c(iii, v)). In addition, Rheb reporter expression was increased by
N1ICD and MAML1 (Fig. 2d(i)), supporting the direct activation of Rheb transcription through NRE2, NRE3, and Notch1.

To determine which promoter elements are essential for the transcriptional activation of Rheb, we altered these potential RBPJ binding sites including NRE2 and NRE3 (one at a time) as previously described ${ }^{43}$. We measured the fold activation of each mutant relative to the activity of the wild-type promoter of Rheb (Fig. 2d(i)). Mutation of the potential RBPJ binding site 1 or 4 did not change the basal and N1ICD-induced promoter activity (Fig. 2d(i)). The mutation in NRE2 or NRE3 significantly suppressed the basal Rheb promoter activity, and the expression of MAML1 and N1ICD was able to raise activity of these mutants only to the level of basal wild-type Rheb promotor (Fig. 2d(i)). The activity of NRE2 and NRE3 double mutant was similar to NRE2 or NRE3 single mutants (Fig. 2d(i)), suggesting that two sites are required for Notch1-dependent transactivation of Rheb and that altering either one is sufficient to eradicate this regulation. These results also suggest that Notch1 regulates approximately $50 \%$ of Rheb promoter basal activity. To confirm that Notch1 transactivates Rheb, we overexpressed N1ICD and MAMl1 or downregulated Notch1 using shRNA in 293T, HeLa, CRL4004, 621-101, and H1299 cells. Overexpression of N1ICD and MAML1 increased the expression of endogenous Rheb by 2 fold on average, whereas downregulation of Notch1 reduced Rheb expression by 5 -fold (Fig. 2d(ii-iv) and Supplementary Fig. 10). Next, we generated focal deletion of the NRE3 region within the endogenous RHEB promoter in CRL4004 angiomyolipoma cells using CRISPR/Cas9. These deletions suppressed expression and activation of Rheb (Supplementary Fig. 4), confirming a direct regulation of Rheb by Notch1.

Our data indicate that Notch1 activates Rheb, and therefore we hypothesized that the oscillation in expression of Rheb (Fig. 2a, b and Supplementary Fig. 10) results from the oscillatory interaction of Notch1 with NRE2 and NRE3. We examined binding of Notch1 to the promoter of Rheb at several time points after cells in DMEM were released from the synchronization conditions $s^{40}$ 41. Notch1 did not bind to the potential RBPJ binding site 1 (Fig. 2e(i) and Supplementary Fig. 8c(i)); in contrast, it bound in an oscillating manner to NRE2 and NRE3 (Fig. 2e-ii-iii and Supplementary Fig. 8C-ii-iii). The level of Notch1 binding (Fig. 2e (ii, iii) and Supplementary Fig. 8C-ii-iii) correlated with the increase in RHEB mRNA levels (Fig. 2a(i)). These data support that NRE2 and NRE3 regulate Rheb expression, in contrast to the potential RBPJ binding site 1, which does not appear to play a role in the regulation of Rheb.

Next, we determined whether the similar mechanism regulates the oscillation of Hes1. We found that Notch1 binds to the Hes1 promoter in an oscillatory manner (Fig. 2e(iv) and Supplementary Fig. 8C-iv), as it was a case for Rheb promoter. Although

Fig. 1 Multipotent progenitor and NSC-like profile of LAM and angiomyolipoma. a Hematoxylin and eosin (H\&E) of the lung sections from sporadic LAM; GFAP or NSE expression in LAM. Arrows indicate LAM and arrowheads normal bronchial mucosa (upper) or normal pleura (lower images). b Sporadic angiomyolipomas (upper left and upper middle) and adjacent normal kidney (upper right) or TSC-associated (lower images) angiomyolipomas, stained with H\&E or for NSE, GFAP, NS-tubulin, or nestin (arrows indicate angiomyolipoma and arrowheads kidney). c (i) GFAP, (ii) NSE and nestin expression in (i) TSC-associated and sporadic (ii) angiomyolipomas ( $T$ ) from patients ( $P$ ) vs. normal kidney (NK) by western immunoblotting. $\mathbf{d}$ (i) Nestin and peripherin expression in LAM (first left) and co-expression analysis by digital pathology; red indicates peripherin (second), brown nestin (third image), and yellow indicates co-expression of both markers (markup, right fifth panel: details within the area marked by the corresponding insert in fourth panel, black arrows). (ii) Quantification for data shown in $\mathbf{d}$ (i) by Aperio Digital Pathology; the percentage of area with co-expression of peripherin and nestin in LAM from 9 patients. Error bars are defined as means + s.e.m. e (i) FACS of cells grown in DMEM medium. Percentages of 621-101 and CRL4004 (angiomyolipomasderived) cells expressing nestin alone or co-expressing: melan-A, GFAP, SMA, and NS-tubulin; (ii) Nestin, GFAP, and NS-tubulin expression in 621-101 (grown in DMEM or IIA complete medium), CRL4004 angiomyolipoma cells, angiomyolipoma tumor, and corresponding normal kidney by western immunoblotting. Error bars are defined as means + s.e.m. f q(RT)-PCR of SOX10, SOX9, ID3, NESTIN, c-Kit, DCT, and S100A1 mRNA levels relative to 185 in 621-101, CRL4004, angiomyolipoma tumor, and corresponding normal kidney. Data represent means ( \pm s.e.m.). Data from nine LAM and eleven angiomyolipomas (a-d). Scale bar: $50 \mu \mathrm{m}$. ${ }^{\star} P \leq 0.05, t$-test. Data are representative of three to six (e (i)) independent experiments 
Hes1 oscillation was shown to be regulated by Notch through use of DAPT ( $\mathrm{N}$-[N-(3,5-difluorophenacetyl)-L-alanyl]-S-phenylglycine $t$-butyl ester $)^{19}$, we additionally report that Hes 1 oscillation occurs by direct cyclic binding of Notch1 to its promoter.

These results suggest that Notch1 regulates Rheb via binding to NRE2 and NRE3. In conjunction with the Rheb ability to activate
Notch $1{ }^{17,18}$, our data also point to a new regulatory circuit involving Notch and Rheb, which we termed the Rheb-Notch-Rheb loop. Since oscillation of Notch maintains multipotent NSCs, we propose that the Rheb-Notch-Rheb loop, controlled by fluctuations in the binding of Notch1 to Rhebactivating and Hes1-activating NREs, contributes to maintaining multipotent properties of angiomyolipoma cells.
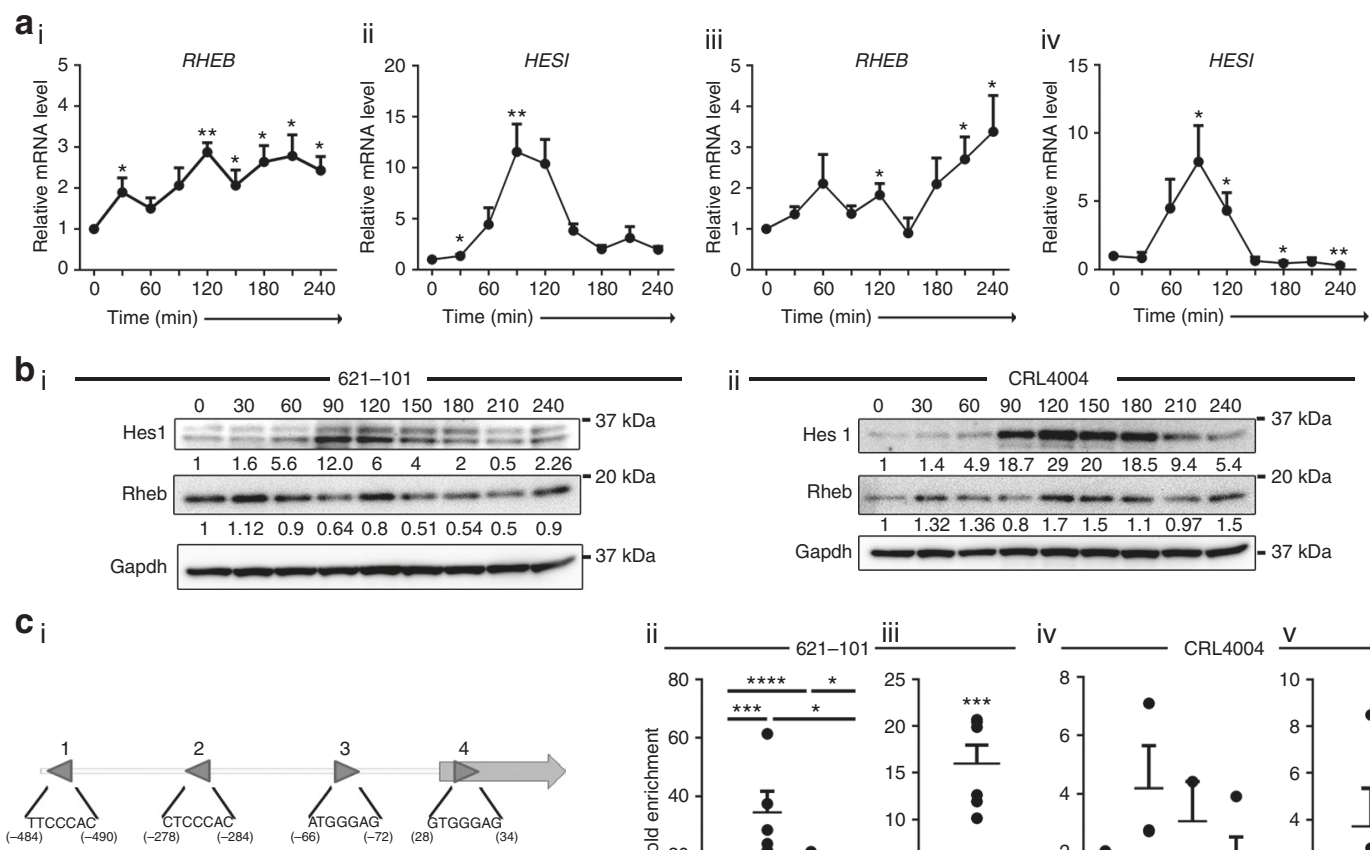

ii
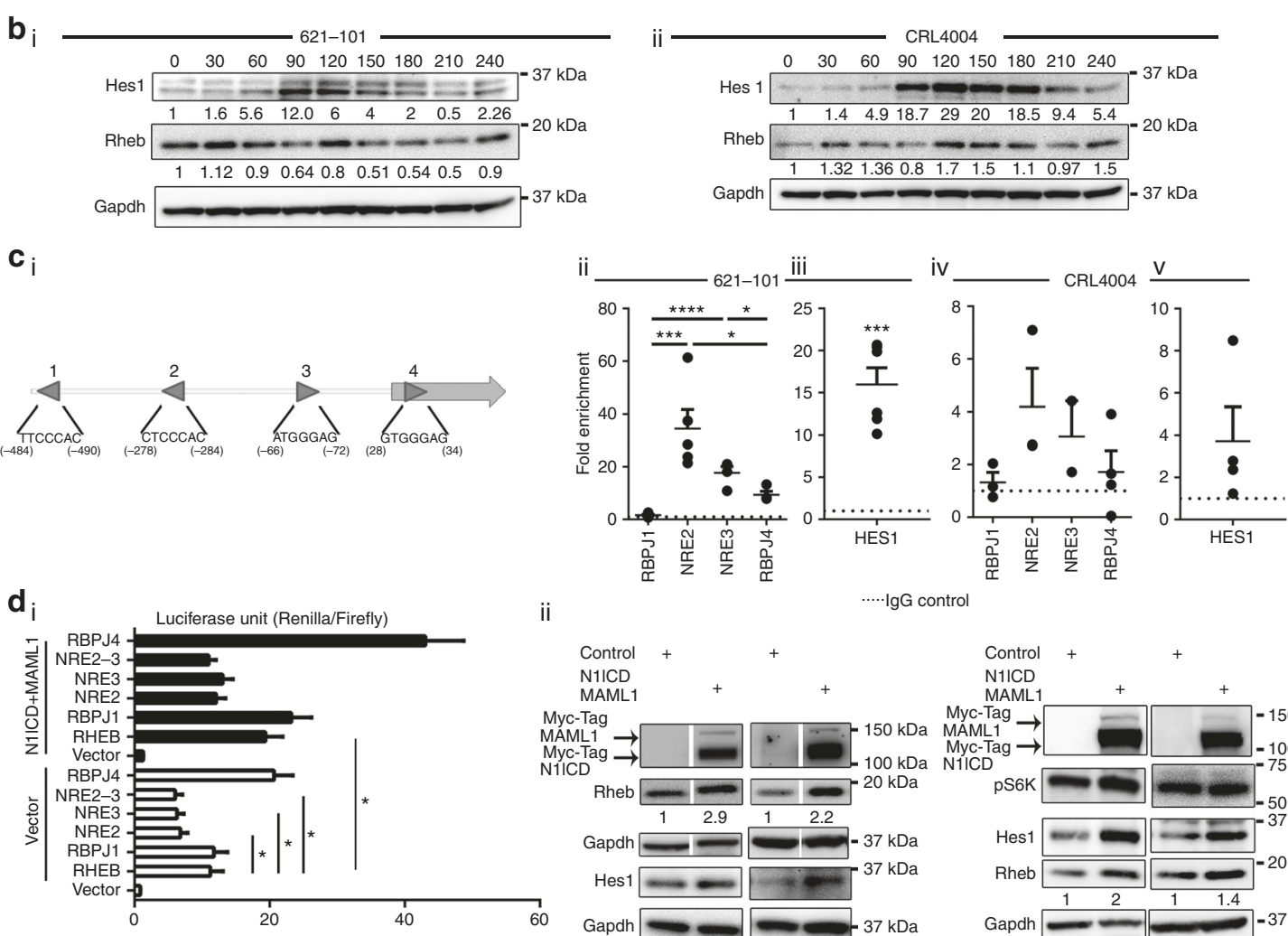

ii

......gG control
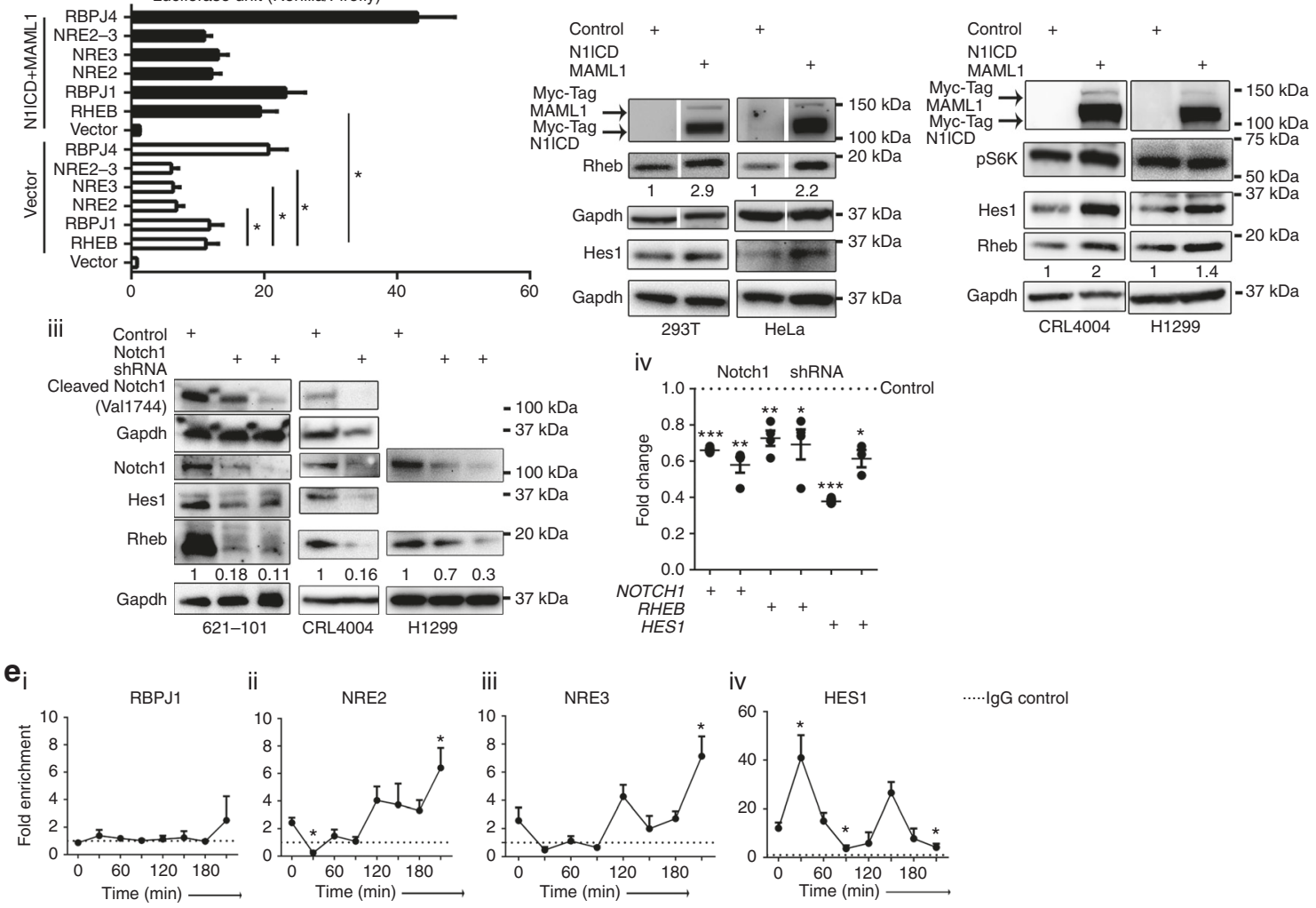

...... IgG control 
Neuronal differentiation of angiomyolipoma cells. Since angiomyolipoma cells express markers of $\mathrm{NC}$ lineages, we examined the ability of these cells to differentiate under conditions favoring glial-neuronal differentiation. We used $\mathrm{N}$-medium promoting differentiation of nestin-positive and carotid bodyderived cells toward glomus cells ${ }^{44}$. In this medium viabilities of angiomyolipoma cells were 79 and $66 \%$ on days 4 and 7 , respectively, and they formed sphere-like structures (Fig. 3a, b, d, e), similar to carotid body stem cell neurospheres ${ }^{4}$. The similar structures were formed by NC-derived melanoma cells (Supplementary Fig. 5D) that express the neuronal marker peripherin ${ }^{45}$.

The loss of nestin in angiomyolipoma cells during the formation of sphere-like structures (Fig. 3b, c(i), e, f(i)) correlated with the adoption of an early neuronal or melanocyte fate, indicated by increases in percentages of NS-tubulin-positive cells (Fig. 3b, c(ii), e, and $\mathrm{f}(\mathrm{ii})$ ) and cells co-expressing NS-tubulin and melan-A (Supplementary Fig. 5E), respectively, suggests neuronal traits. The adoption of these fates correlated with an exit from the cell cycle, indicated by the decreased number of Ki-67-positive cells (Fig. 3c (iii), $\mathrm{f}(\mathrm{iii})$ ), and the induction of neuronal (Ngn1) and melanocytic (DCT and/or $c$-Kit) differentiation genes (Fig. 3c(iv), f(iv)).

To determine whether hyperactivation of Rheb and Notch prevents neuronal differentiation, we used short hairpin RNA (shRNA) to deplete angiomyolipoma cells of Rheb ${ }^{46}$ or Notch1. This depletion accelerated the formation of neural-like spheres (Supplementary Fig. 5F-i, G-i) and differentiation along the neuronal lineage in $\mathrm{N}$-medium, demonstrated by faster acquisition of NS-tubulin (Supplementary Fig. 5F-ii, G-ii). In contrast, overexpression of the constitutively active form of Rheb $(\mathrm{Q} 64 \mathrm{~L})^{47}$ blocked neuronal differentiation of angiomyolipoma cells in $\mathrm{N}$ medium, as the number of NS-tubulin-positive cells was reduced by 2 -fold (Supplementary Fig. $5 \mathrm{H}$ ). Of note, transient overexpression of Rheb does not allow for prolonged (up to 7 days) follow-up of cell phenotypes due to lack of chromosomal plasmid integration. Therefore, this experiment involved only $24 \mathrm{~h}$ of exposure to $\mathrm{N}$-medium, after $24 \mathrm{~h}$ of transfection ( $48 \mathrm{~h}$ total). The differentiation along the neuronal or melanocyte lineage was not affected by rapamycin (Supplementary Fig. 5I) suggesting again a distinct role of the Rheb-Notch pathway in this process, independent from mTOR signaling.

Rheb-Notch-Rheb loop in angiomyolipoma differentiation. We hypothesized that alterations in RHEB and HES1 expression are required for neuronal differentiation of angiomyolipoma cells. Therefore, we examined expression of RHEB and HES1 mRNA and binding of Notch1 to NREs within the Rheb and Hes1 promoters in $\mathrm{N}$-medium. Relative to DMEM, N-medium repressed the expression of both RHEB and HES1 mRNA and block oscillation (Fig. 4a(i, ii), Supplementary Fig. 6A-B and
Supplementary Fig. 8A-B). This effect was present in synchronized cells regardless of type of stimulation, serum (Fig. 4a, green lines), or chick embryo extract (Fig. 4a, Supplementary Fig. 6A-B, red lines, and Supplementary Fig. 8A-B, red dots). ChIP-qPCR revealed that in conditions favoring neuronal differentiation $(\mathrm{N}$ medium), binding of Notch1 to NRE2 and NRE3 on the Rheb promoter was significantly reduced compared to DMEM (Fig. $4 \mathrm{~b}$ (i, ii) and Supplementary Fig. 8C). We did not detect significant binding of Notch1 to the potential RBPJ binding site 1 in Nmedium (Fig. $4 \mathrm{~b}(\mathrm{ii}))$. The binding of Notch1 to the Hes1 promoter was also reduced in N-medium (Fig. $4 \mathrm{~b}$ (iii)). WB and qPCR confirmed that N1ICD, Rheb, and Notch target gene HES5 were reduced in angiomyolipoma cells in N-medium (Fig. 4c, d, f, $\mathrm{g}$ and Supplementary Fig. 10). The suppression of Rheb was also demonstrated by reductions in the percentage of phospho-S6positive cells (Fig. 4e, h), reduced phospho-S6-K (Fig. 4g and Supplementary Fig. 10), and decreased expression of Rheb (Fig. 4f, g and Supplementary Fig. 10). The neuronal differentiation of angiomyolipoma cells was associated with reduced SOX9 and IDI and ID3 (Fig. 4i), consistent with the role of Notch in the activation of these genes ${ }^{48}$, a role for SOX9 in maintaining multipotent $\mathrm{NSCs}^{49}$, and Id proteins in preventing neurogenesis in neural tube $(\mathrm{NT})^{50,51}$.

We propose that decreased binding of Notch1 to NREs may lead to suppression of Rheb and Hes1 expression and decreased oscillation, leading to sustained, low level of Rheb and Hes1, followed by neuronal differentiation of angiomyolipoma cells. Given that the suppression of Rheb correlated with the suppression of Notch1 during neuronal differentiation of angiomyolipoma cells, we conclude that inhibition of the RhebNotch-Rheb regulatory loop is required for this process. Conversely, ongoing activity of this loop maintains multipotent properties of angiomyolipoma cells in DMEM.

The loss of Tsc1 delays the differentiation of NSCs in vivo. The differentiation abnormalities in murine Tsc-null neuroepithelial progenitors were reported ${ }^{52,53}$. Therefore, we next determined whether the loss of $T s c 1 / 2$ blocks progenitor differentiation during mouse embryogenesis via a mechanism similar to that in angiomyolipoma cells. We used a genetic strategy for cell-specific depletion of $T s c 1$ in the mouse embryo NT because expression of ID1 and ID3 was suppressed in angiomyolipoma cells in Nmedium (Fig. 4i), Id proteins as well as high level of Hes1 prevent neurogenesis in $\mathrm{NT}^{50,51}$, and Id 3 and Hes1 are regulated by Notch $1^{54}$. We utilized lineage tracing in $R O S A^{m T / m G}$ mice (Fig. 5) with a floxed tdTomato (mT)-cassette and a stop codon upstream of the EGFP (mG)-cassette (Fig. 5a, b and Supplementary Fig. 7A-C). This system along with Cre strains allows comparison of the control- and mutant-EGFP ${ }^{+}$cells. We employed a nestin or

Fig. 2 Notch1 directly activates the Rheb promoter. a q(RT)-PCR of (i, iii) RHEB and (ii, iv) HES1 relative to GAPDH in synchronized (i, ii) 621-101 and (iii, iv) CRL4004 angiomyolipoma cells in DMEM. The statistical significance of RHEB and HES1 expression at different time points was determined relative to time 0. b Expression of Rheb and Hes1 in synchronized (i) 621-101 and (ii) CRL4004 angiomyolipoma cells in DMEM by western immunoblotting. Numeric values represent densitometry analysis of the expression of Rheb relative to expression of Gapdh. c (i) Rheb promoter: triangles indicate potential RBPJ binding sites with a reverse $(1,2)$ and a forward $(3,4)$ orientation. (ii-v) Binding of Notch1 to (ii, iv) the potential RBPJ binding site 1 , NRE2, NRE3, and the potential RBPJ binding site 4 of RHEB or (iii, v) HES1 promoter in non-synchronized (ii, iii) 621-101 or (iv, v) CRL4004 cells grown in DMEM by ChIP-qPCR; binding is shown as fold enrichment over the lgG (dotted line). d (i) Wild-type Rheb-luciferase (RHEB), RHEB-RBPJ1- (RBPJ1), RHEB-NRE2- (NRE2), RHEB-NRE3- (NRE3), or RHEB- RBPJ4- (RBPJ4) mutant-luciferase or control-luciferase (control) promoters activity in HeLa cells. (ii) Expression of N1ICD, MAML1, Hes1, Rheb, and phospho-S6K in 293T, HeLa, CRL4004, and H1299 cells. (iii) Expression of cleaved Notch1 (Val1744), Notch1, Hes1, Rheb in 621-101, CRL4004, and H1299 cells depleted of Notch1 using shRNA by western blotting or (iv) q(RT)-PCR. Numeric values represent densitometry analysis of the expression of Rheb relative to expression of Gapdh. (iv) NOTCH1, RHEB, and HES1 relative to GAPDH in 621-101 cells depleted of Notch1 using shRNA. e Binding of Notch1 to the (i) potential RBPJ1 site, (ii) NRE2, and (iii) NRE3 within Rheb or (iv) Hes1 promoter in synchronized 621-101 cells in DMEM by ChIP-qPCR as in (c (ii, iii)). Data represent means \pm s.e.m. Error bars are defined as means + s.e.m. ${ }^{\star} P \leq 0.05,{ }^{\star \star} P \leq 0.01,{ }^{\star \star \star} P \leq 0.001, t$-test. Data are representative of three (a (iii, iv), b, d (i-iv)), four (e), and six (a (i, ii), c (ii, iii)) independent experiments 
a

621-101

b
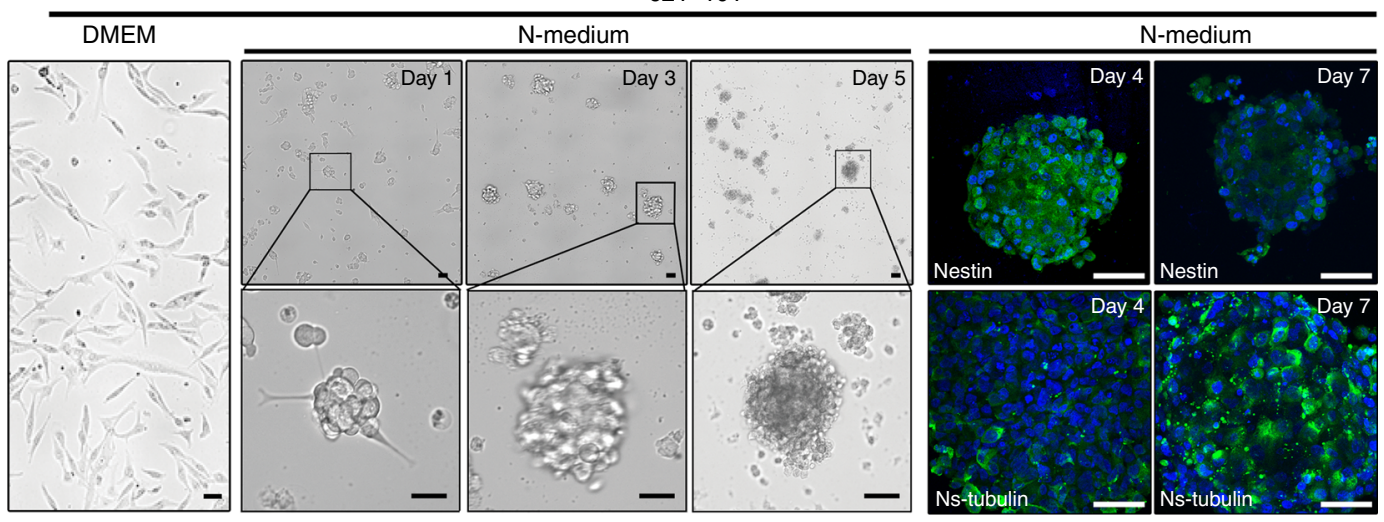

$\mathbf{C}_{\mathbf{i}}$

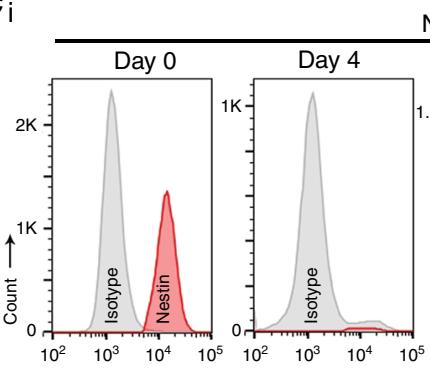

Nestin
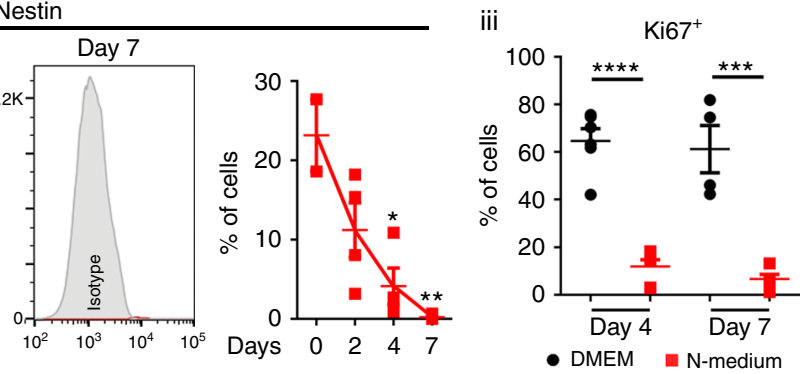

ii

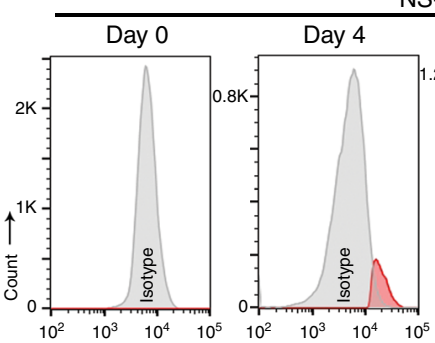

NS-tubulin
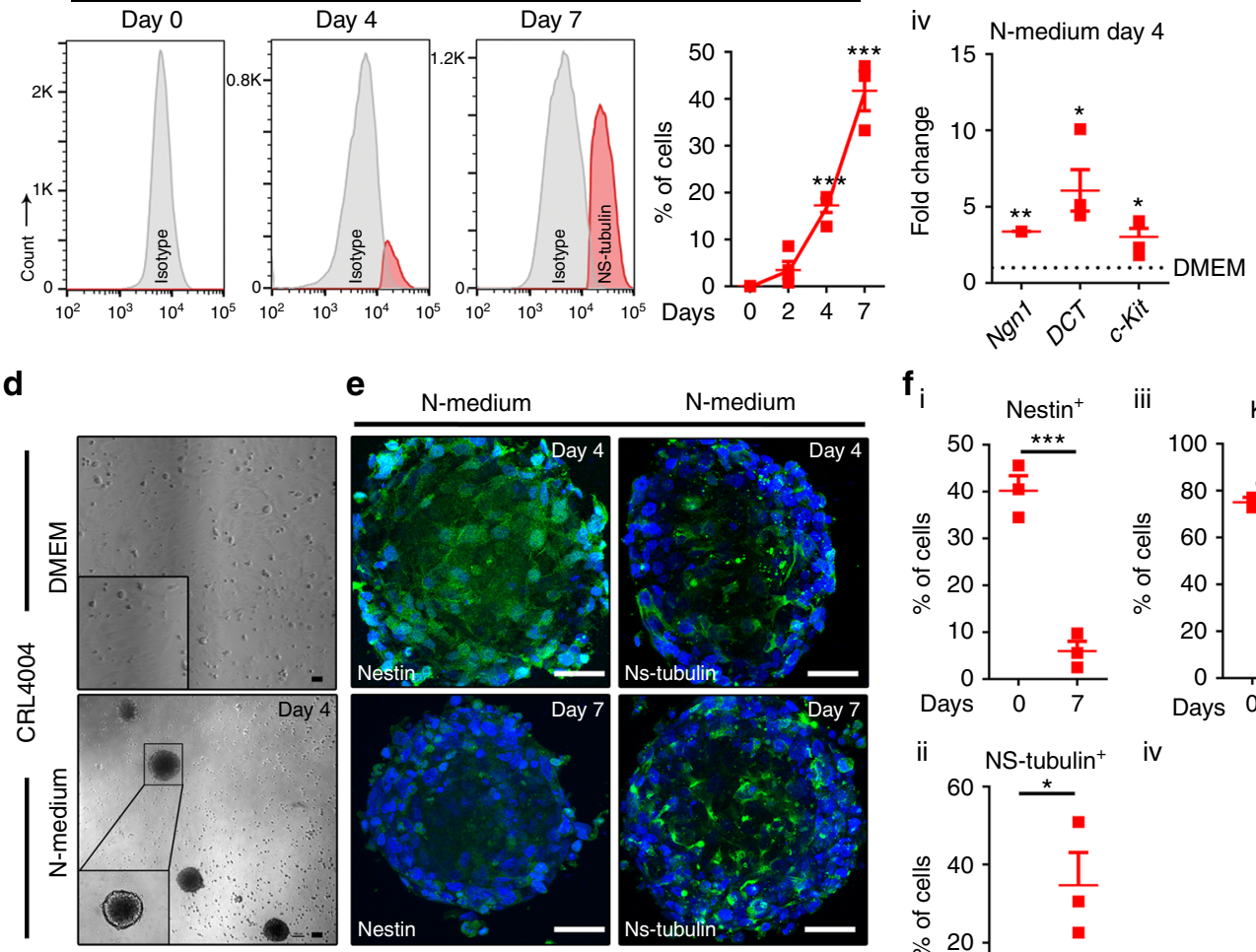

$\mathrm{N}$-medium

$\mathbf{f}_{\mathrm{i}}$
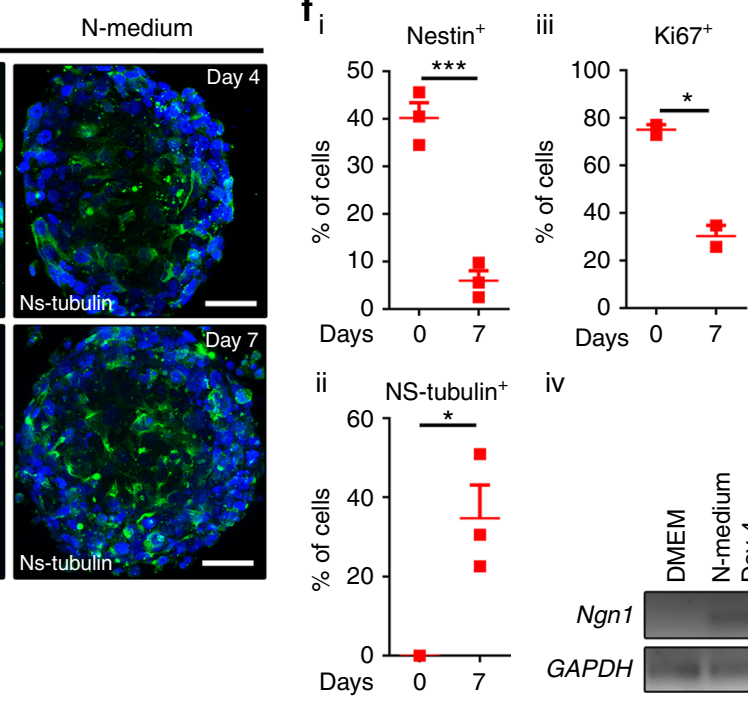

iv

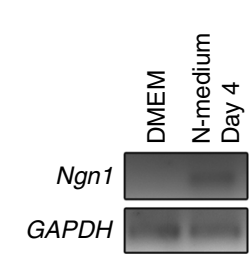

Fig. 3 Neuronal differentiation of angiomyolipoma cells. a, d Brightfield images of $\mathbf{a}$ 621-101 and d CRL4004 cells in DMEM or N-medium. b, e Nestin and NS-tubulin in b 621-101 and e CRL4004 sphere-like structures in N-medium at day 4 and day 7 by immunofluorescence. c, $\mathbf{f}$ FACS of $\mathbf{c}$ 621-101 and $\mathbf{f}$ CRL4004 cells in N-medium. c, $\mathbf{f}$ Representative histograms and graphs showing percentages of cells in N-medium expressing (c (i), $\mathbf{f}$ (i)) nestin only and (c (ii), f (ii)) NS-tubulin only. c (iii), f (iii) Ki-67 in (c (iii)) 621-101 and (f (iii)) CRL4004 cells in N-medium vs. DMEM at days 4 and 7. c (iv), f (iv) $\mathrm{q}(\mathrm{RT}$ )-PCR of Ngn1, DCT, and c-Kit mRNA relative to GAPDH in c (iv) 621-101 or f (iv) CRL4004 cells in N-medium vs. DMEM at day 4. Data represent means \pm s.e.m. Error bars are defined as means + s.e.m. ${ }^{\star} P \leq 0.05,{ }^{\star \star} P \leq 0.01,{ }^{\star \star \star} P \leq 0.001,{ }^{\star \star \star \star} P \leq 0.0001$ by $t$-test. Data are representative of two (b, e), three, $n=3$ (f (i-iv), c(iv)), four (d); $n=4$ (c (i-iii)), and seven (a) independent experiments 
$\mathbf{a}_{\mathrm{i}}$

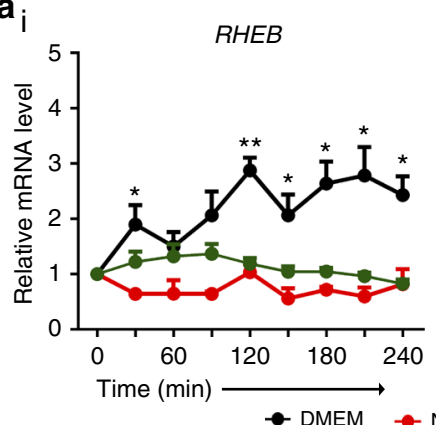

ii HES1

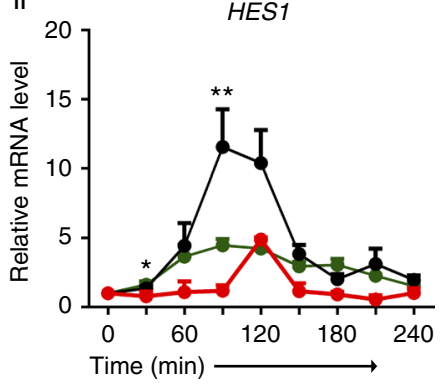

$\mathbf{b}_{\mathrm{i}}$
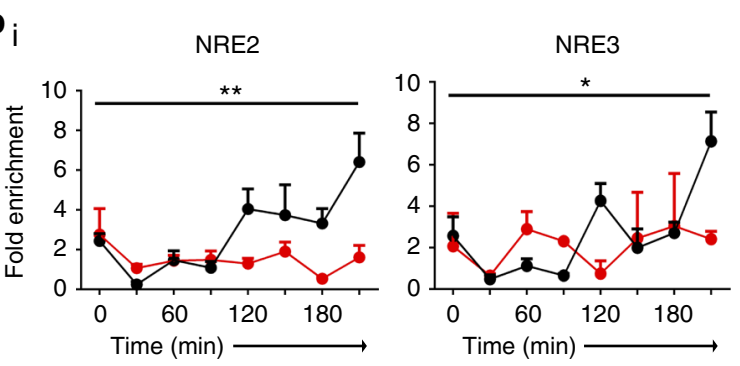

ii

RBPJ1

iii
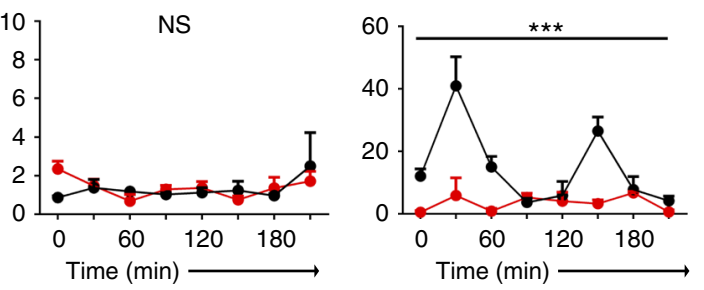

- DMEM $\rightarrow \mathrm{N}$-medium Day 2 …..IgG control

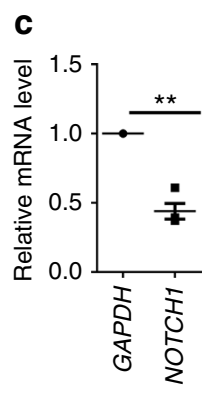

d

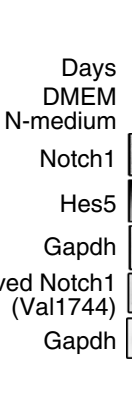

g

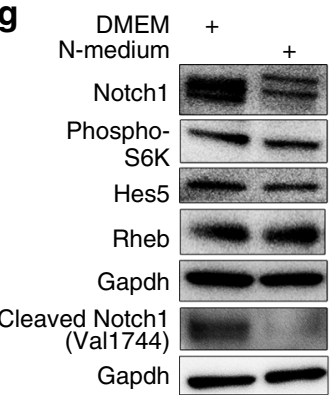

Day 1

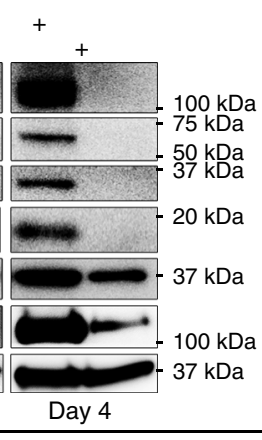

Day 4

CRL4004

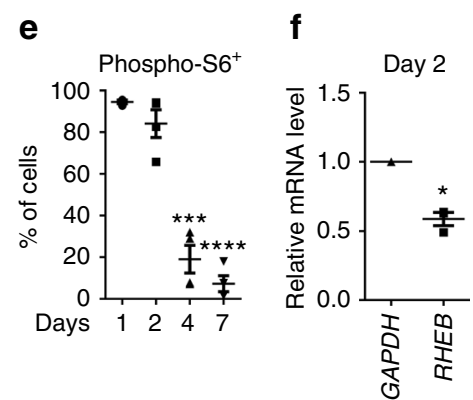

h i

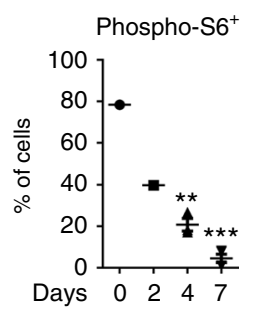

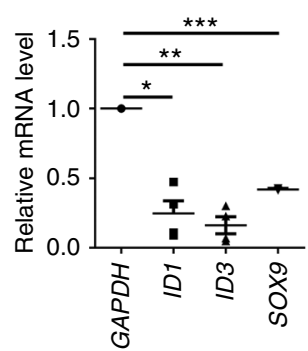

Fig. 4 Suppression of Rheb and Hes1 oscillation associates with neuronal differentiation of angiomyolipoma cells. a q(RT)-PCR of (i) RHEB and (ii) HES1 relative to GAPDH in synchronized 621-101 cells in N-medium (released with chicken embryo extract (red lines) or serum (green lines)) vs. DMEM (released with serum (black lines)) (data from DMEM are also used in Fig. 2a (i-ii)). The statistical significance of RHEB and HES1 expression in DMEM vs. $\mathrm{N}$-medium was determined between corresponding time points. b Binding of Notch1 to the (i) NRE2, NRE3, and (ii) potential RBPJ1 site within the Rheb or (iii) Hes1 promoter, in synchronized 621-101 cells in N-medium vs. DMEM at day 2 by ChIP-qPCR (data from DMEM are also used in Fig. 2e (i-iv)). Asterisks represent the statistical significance of mean of time points in DMEM vs. N-medium, two-way ANOVA. c q(RT)-PCR of NOTCH1 in 621-101 cells in N-medium at day 2, relative to DMEM (control) after normalization to GAPDH. d Western blot of Notch1, Hes5, and cleaved Notch1 (Val1744) in 621-101 cells in N-medium vs. DMEM. e Percentage of 621-101 cells expressing phospho-S6 in N-medium by FACS. f q(RT)-PCR of RHEB in 621-101 cells in $\mathrm{N}$-medium at day 2, relative to DMEM (control) after normalization to GAPDH. $\mathbf{g}$ Western blot of Notch1, phospho-S6K, Hes5, Rheb, and cleaved Notch1 (Val1744) in CRL4004 cells in N-medium vs. DMEM. h Percentage of CRL4004 cells expressing phospho-S6 grown in N-medium by FACS. $\mathbf{i}$ q(RT)-PCR of ID1, ID3, and SOX9 in 621-101 cells in N-medium at day 2, relative to DMEM (control) after normalization to GAPDH. Data represent means \pm s.e.m. Error bars are defined as means + s.e.m. ${ }^{\star} P \leq 0.05,{ }^{\star \star} P \leq 0.01,{ }^{\star \star \star} P \leq 0.001,{ }^{\star \star \star \star} P \leq 0.0001, t$-test $(\mathbf{a}, \mathbf{c}, \mathbf{e}, \mathbf{f}, \mathbf{h}, \mathbf{i})$, two-way ANOVA (b). Data are representative of two, $n=2$ (i for SOX9); three (g); three, $n=2$ (a, b (iii)); three, $n=3$ (h); three, $n=4$ (b (i, ii)); four (d), four, $n=3(\mathbf{f}) ;$ four, $n=4$ (c, e, i for ID1, ID3) 
a
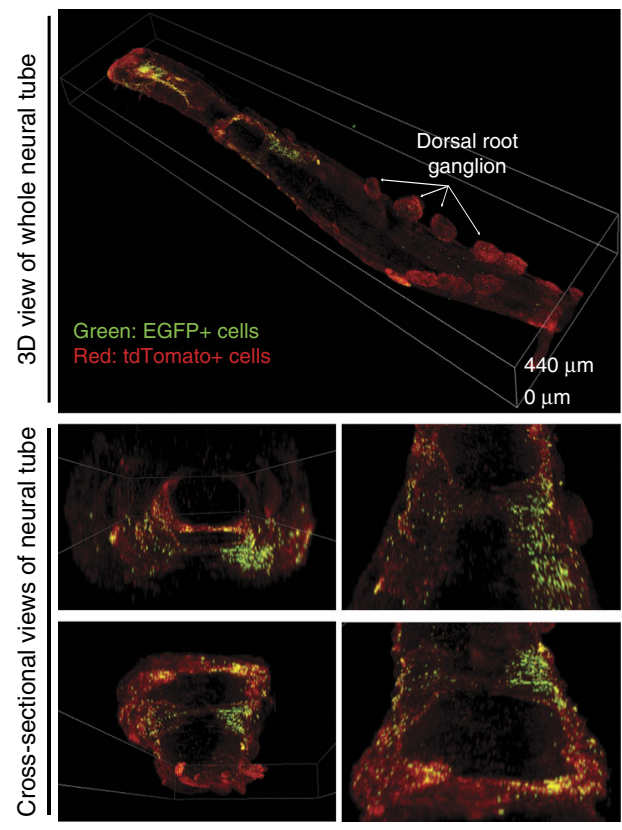

b

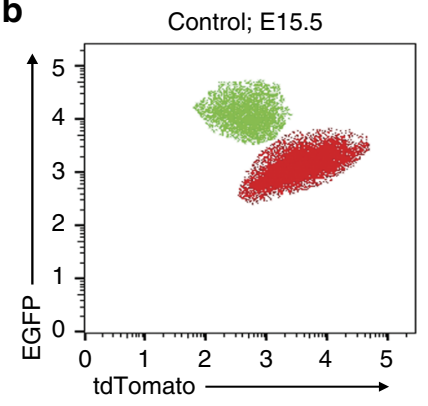

GFAP-Cre; ROSA ${ }^{m T / m G}$

; $T s c 1^{\text {tf }}$; E15.5

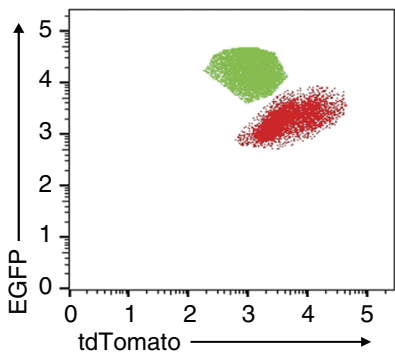

iii Nestin $^{+} \mathrm{GFAP}^{+}$

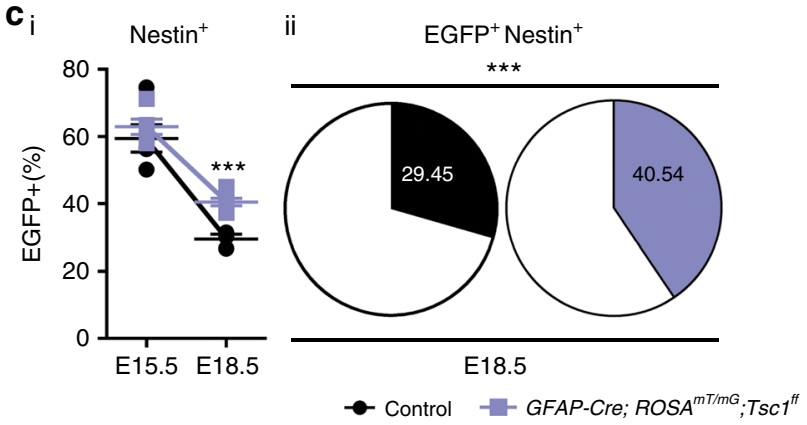

$\mathbf{d}_{\mathrm{i}}$

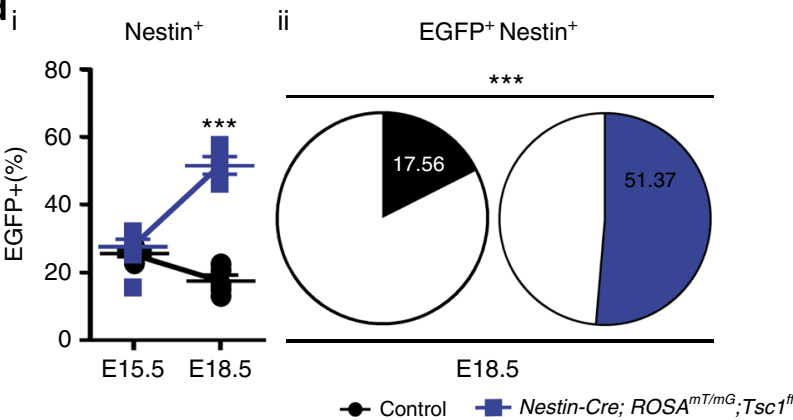

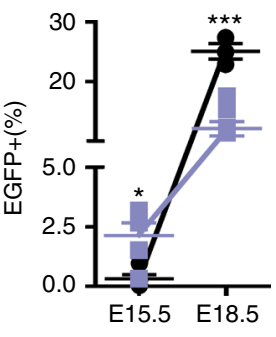

iv GFAP $^{+}$

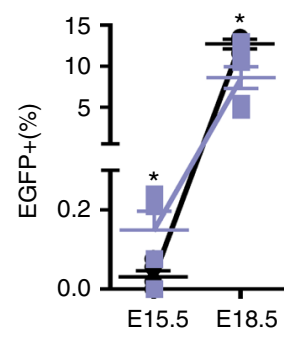

iii

Nestin $^{+}$GFAP $^{+}$

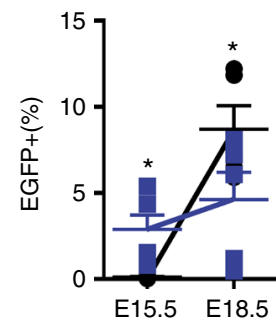

GFAP $^{+}$

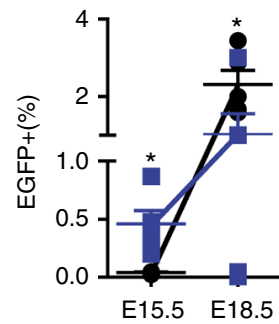

Fig. 5 Loss of Tsc1 delays the differentiation of NSCs and RGCs. a Confocal microscopy of EGFP-positive (EGFP ${ }^{+}$) and tdTomato-positive (tdTomato ${ }^{+}$) NT cells in a ROSA ${ }^{m T / m G}$ mouse NT. Maximum intensity projections from Z-stacks is shown. b-d FACS of EGFP ${ }^{+}$NT cells in b, c GFAP-Cre;ROSA ${ }^{m T / m G}$ (control) vs. GFAP-Cre;ROSA ${ }^{m T / m G}$;Tsclff embryos or d Nestin-Cre;ROSA ${ }^{m T / m G}$ (control) vs. Nestin-Cre;ROSA ${ }^{m T / m G} ;$ Tsc ff embryos. b Representative FACS plots of EGFP ${ }^{+}$or tdTomato ${ }^{+}$NT cells. c, d GFAP-Cre;ROSA ${ }^{\mathrm{mT} / \mathrm{mG}}$ (control-black) vs. GFAP-Cre;ROSA ${ }^{\mathrm{mT} / \mathrm{mG}}$;Tsciff embryos (blue) (c) or Nestin-Cre;ROSA ${ }^{\mathrm{mT} /}$ $m G$ (control-black) vs. Nestin-Cre;ROSA ${ }^{m T / m G} ;$ Tsciff embryos (blue) (d). (i) Change in the percentages of EGFP ${ }^{+}$nestin ${ }^{+} \mathrm{NT}$ cells from E15.5 to E18.5 embryos. (ii) Charts representing EGFP ${ }^{+}$nestin ${ }^{+}$NT cells at E18.5. (iii, iv) Change in the percentages of (iii) EGFP ${ }^{+}$nestin ${ }^{+} G_{F A P^{+}}$or (iv) EGFP ${ }^{+} \mathrm{GFAP}^{+}$ NT cells from E15.5 to E18.5 embryos. Data represent means \pm s.e.m.. Error bars are defined as means + s.e.m. ${ }^{\star} P \leq 0.05,{ }^{\star \star \star} P \leq 0.001 t$-test. Data are representative of two experiments, $\mathrm{n}_{\text {GFAP-Cre;ROSAmT/mG }}($ E15.5 $n=5$, E18.5 $n=3), \mathrm{n}_{\text {GFAP-Cre;ROSAmT/mG;Tsclff }}($ E15.5 $n=5$, E18.5 $n=7)\left(\mathbf{c}\right.$ (i-iv)); $\mathrm{n}_{\text {Nestin-Cre; }}$ ROSAmT/mG $(E 15.5 n=4$, E18.5 $n=5), \mathrm{n}_{\text {Nestin-Cre;ROSAmT/mG;Tsclff }}(E 15.5 n=7$, E18.5 $n=5)(\mathbf{d}(\mathrm{i}, \mathrm{ii})) ; \mathrm{n}_{\text {Nestin-Cre;ROSAmT/mG }}($ E15.5 $n=4$, E18.5 $n=5), \mathrm{n}_{\text {Nestin- }}$ Cre;ROSAmT/mG;Tsclff (E15.5 $n=6$, E18.5 $n=5$ ) (d (iii, iv)) embryos

GFAP-Cre driver to remove the floxed Tsc1 allele from the NSCs and early and late neuronal progenitors, respectively, since both promoters are active in LAM and angiomyolipoma (Fig. 1). We compared control- and mutant-EGFP ${ }^{+}$cells: (1) nestin-positive cells, representing NSCs ${ }^{23,55}$; (2) nestin- and GFAP-expressing cells (radial glial progenitors (RGCs) $)^{23}, 55,56 ;(3)$ nestin- and NS-tubulin-expressing cells (neuronal progenitors) ${ }^{57}$; (4) GFAPexpressing cells (glial precursors); and (5) NS-tubulin-expressing cells (differentiated neurons) (Fig. 5, Supplementary Fig. 7A-C, and Supplementary Table 4). 
As embryos matured (E15.5 vs. E18.5), the number of NSCs decreased, as expected, in both control strains (Fig. 5c(i), d(i) and Supplementary Table 4). In GFAP-Cre;ROSA ${ }^{m T / m G} ; T s c 1$ ff mice, we observed a slower reduction in the number of Tsc1-null NSCs $\left(\right.$ EGFP $^{+}$nestin $^{+}$) (Fig. 5c(i) and Supplementary Table 4), indicated by the higher number, compared to the control (wild-type), of NSCs at E18.5 (Fig. 5c(ii), Supplementary Fig. 7B, and Supplementary Table 4). In Nestin-Cre;ROSA ${ }^{m T / m G}$; Tsc $1^{f f}$ embryos, the Tsc1-null NSCs accumulated with time, with their number tripling by E18.5 (Fig. 5d(i, ii), Supplementary Fig. 7C, and Supplementary Table 4). As an embryo matures, NSCs give rise to the RGCs, which then differentiate along neuronal and glial lineages ${ }^{58}$. Therefore, we examined the rate of differentiation of RGCs and glial precursors.

RGCs $\left(\mathrm{EGFP}^{+}\right.$nestin $^{+} \mathrm{GFAP}^{+}$) in control mice (GFAP-Cre; $R_{O S A^{m T / m G}}$ and Nestin-Cre;ROS $A^{m T / m G}$ ) increased over time by 15- and 60-fold, respectively (Fig. $5 \mathrm{c}$ (iii), d(iii) and Supplementary Table 4), indicating the differentiation of wild-type NSCs toward RGCs. However, in GFAP-Cre;ROSA ${ }^{m T / m G} ; T s c l_{f f}$ and Nestin-Cre; $R O S A^{m T / m G} ;$ Tscl $1^{f f}$ embryos, RGCs increased by only $3.7-$ and 1.6fold, respectively, over time (Fig. $5 c$ (iii), d(iii) and Supplementary Table 4). A similar reduction in differentiation toward glial precursors $\left(\mathrm{EGFP}^{+} \mathrm{GFAP}^{+}\right.$) occurred in the Tsc-1-null EGFP ${ }^{+} \mathrm{GFAP}^{+}$cells, compared to the controls (Fig. $5 \mathrm{c}$ (iv), d(iv) and Supplementary Table 4). The slower rate of differentiation along the RGC and glial lineages, in conjunction with the increased number of Tsc-1-null NSCs at E18.5, suggests impairment in the Tsc-1-null NSC differentiation toward these lineages.

Oscillation of Hes1 and Rheb blocks NSC differentiation. Because Tsc1 loss led to accumulation of NSCs, we next sought to determine whether Tsc1 regulates Notch oscillation and expression of Rheb in NSCs in vivo. The NSCs $\left(\right.$ nestin $^{+}$) were isolated from E15.5 Nestin-Cre;Tsclff and littermate control mouse embryo NTs (Supplementary Fig. 7D, E-i), synchronized by serum starvation, and evaluated for Rheb and Hes1 mRNAs. The analysis of repeated measures demonstrated that Hes 1 oscillation patterns differ significantly $(P=0.0146$ by regression analysis of longitudinal data) between controls vs. mutant mice, with higher amplitudes of the oscillations in Tsc1-null cells. The changes in Rheb mRNA expression correlated positively with the Hes1 oscillations in control (Fig.6a-i, iii, $R^{2}=0.579, P<0.0001$ by regression analysis of longitudinal data) and Tsc1-null NSCs (Fig. 6a-ii-iv, $R^{2}=0.36, P=0.02$ by regression analysis of longitudinal data). The mean expression of averaged Rheb and Hes 1 was significantly different in Nestin-Cre; Tsclff embryos vs. wildtype littermate controls $(P=0.0201$ by regression analysis of longitudinal data). Gapdh levels did not change over time as indicated by raw CT values for each time point (Supplementary Fig. 7F). Therefore, we concluded that Tscl regulates Notch and, subsequently, Rheb oscillation. WB and densitometry analyses confirmed increased oscillation of Rheb and Hes1 over time in Tsc1-null NT cells compared to NT cells of littermate controls (Fig. 6c(i) and Supplementary Fig. 10), which was indicated by 5 fold vs. 1.5-fold increase in the expression of Rheb at $120 \mathrm{~min}$ relative to $0 \mathrm{~min}$ in NT cells of Tsc1-null and littermate control embryos, respectively (Fig. 6c(i) and Supplementary Fig. 10). Furthermore, immunofluorescence (IF) analysis demonstrated higher expression of Hes1 in Tsc1-null NT cells and a 'salt-andpepper' pattern of staining (Supplementary Fig. 7G) indicative for Hes1 oscillation ${ }^{19}$. The partial loss of Notch1 in synchronized E15.5 Tsc1-null neuronal progenitors (Nestin-Cre;Tsc1 ${ }_{f} ; \mathrm{Notch}^{f+}$ ) (Supplementary Fig. 7E-ii) reduced significantly $(P=0.0054$ of averaged Rheb and Hes 1 by regression analysis of longitudinal data) oscillation of Rheb and Hes1 mRNAs compared to the wild- type littermate controls, ultimately leading to low and sustained expression of Rheb (Fig. 6b). WB blotting and densitometry analyses confirmed that partial loss of Notch1 function suppresses Rheb and Hes1 oscillation and expression in NT cells (Fig. 6c(ii) and Supplementary Fig. 10). Next, NTs from Nestin-Cre;Tsc1ff, Nestin-Cre; Tsclff; Notch $^{f+}$, and littermate controls were dissected, dissociated, and subjected to FACS. The NSCs containing pS6 were examined, since pS6 serves as an indicator of mTOR hyperactivation, resulting from the loss of Tsc1 (Fig. 6d(i)). The loss of Tsc1 increased numbers of $\mathrm{pS}^{+}{ }^{+} \mathrm{Nestin}{ }^{+} \mathrm{NSC}$ within the NTs of Nestin-Cre;Tsclff embryos (Fig. 6d(ii)). The partial loss of Notch1 in Tsc1-null embryos (Nestin-Cre;Tsc1 ff; Notch $^{f+}$ ) (Supplementary Fig. $7 \mathrm{H}$ ) reduced the number of $\mathrm{pS}^{+}$cells (Fig. 6d(i)) and pS6 $^{+}$NSCs (Fig. 6d(ii)), which is consistent with Notchdependent suppression of Rheb. These results were confirmed using ROS $A^{m T / m G}$ mice. The partial loss of Notch1 in Tsc1-null embryos (Nestin-Cre;ROSA ${ }^{m T / m G}$; Tsclff; ${ }_{\text {Notch }}^{f+}$ ) reduced NSCs when compared to Tsc1-null embryos with intact Notch1 alleles (Nestin-Cre;ROSA ${ }^{m T / m G} ;$ Tsc1ff) (Fig. 6d(iii), Supplementary Fig. 7I). In summary, data support a role of the Rheb-Notch-Rheb loop in maintaining NSCs during mouse embryogenesis because disruption of this loop by removing one allele of Notch suppressed the oscillation of Hes1 and Rheb (Fig. 6b, c(ii) and Supplementary Fig. 10) and prevented the accumulation of NSCs caused by the loss of Tsc1 (6d(ii, iii)).

The Rheb/Notch/Rheb loop operates in Tsc-null tumors. We utilized uterine leiomyoma-derived Tsc2-null ELT3 cells ${ }^{59}$, since the majority of these cells expressed nestin alone (Fig. 6e(i)). WB confirmed expression of nestin in ELT3 cells (Fig. 6e-ii and Supplementary Fig. 10). We used these cells in a xenograft model $^{17}$ to determine the role of the Rheb-Notch-Rheb loop in TSC tumorigenesis. qPCR of the ELT3 tumors revealed a positive correlation between Rheb and Hes 1 mRNAs (Supplementary Fig. 7J), which is associated with the high number of nestinpositive cells within these tumors (Fig. 6f(i)-ii, placebo). The experimental disruption of the Rheb-Notch-Rheb loop by DAPT affected the multipotency of the cells because the number of nestin $^{+}$Tsc2-null cells (Fig. $6 \mathrm{f}(\mathrm{i}, \mathrm{ii})$ ) and Rheb mRNA and protein in these tumors (Fig. 6f(iii-v) and Supplementary Fig. 10) were reduced. This was associated with reduction of Tsc2-null tumor growth (Supplementary Fig. 7K), consistent with previous reports ${ }^{17,18}$. DAPT inhibition of Notch signaling was verified by immunoblotting (Fig. 6f(iv) and Supplementary Fig. 10).

The cell-specific $T s c 1$ loss leads to renal carcinoma. The cellspecific depletion of $T s c 1$ in nestin-expressing kidney cells in adult Nestin-TamCre;Tsc1 f/f mice resulted in multiple bilateral cysts, renal intraepithelial neoplasia (RIN), and invasive multifocal papillary carcinomas composed of eosinophilic tumor cells (Fig. 7b, c), consistent with morphology observed in TSCassociated $\mathrm{RCCs}^{60}$. The origin of renal lesions from nestinexpressing progenitors is suggested by the single nestin-positive cells, containing pS6, which have spindle-shape morphology, similar to these progenitors, within the malignant renal lesions (Fig. 7d-g and Supplementary Fig. 9, arrows: nestin [red], pS6 [green], arrowheads: co-localization of pS6 and nestin). We also determined that renal lesions express Cre recombinase (Fig. 7h) confirming that observed phenotype is linked to Cre recombinase-driven targeting of Tsc1. Cre recombinase is expressed at high levels within tumor cells and dysplastic tubules adjacent to papillary neoplastic lesion, indicating that tumor and dysplastic cells origin from Cre-expressing progenitors (Fig. 7h). The less evident expression of Cre in intact glomeruli (Fig. 7h, upper right corner of the image) is expected, as podocytes and the 


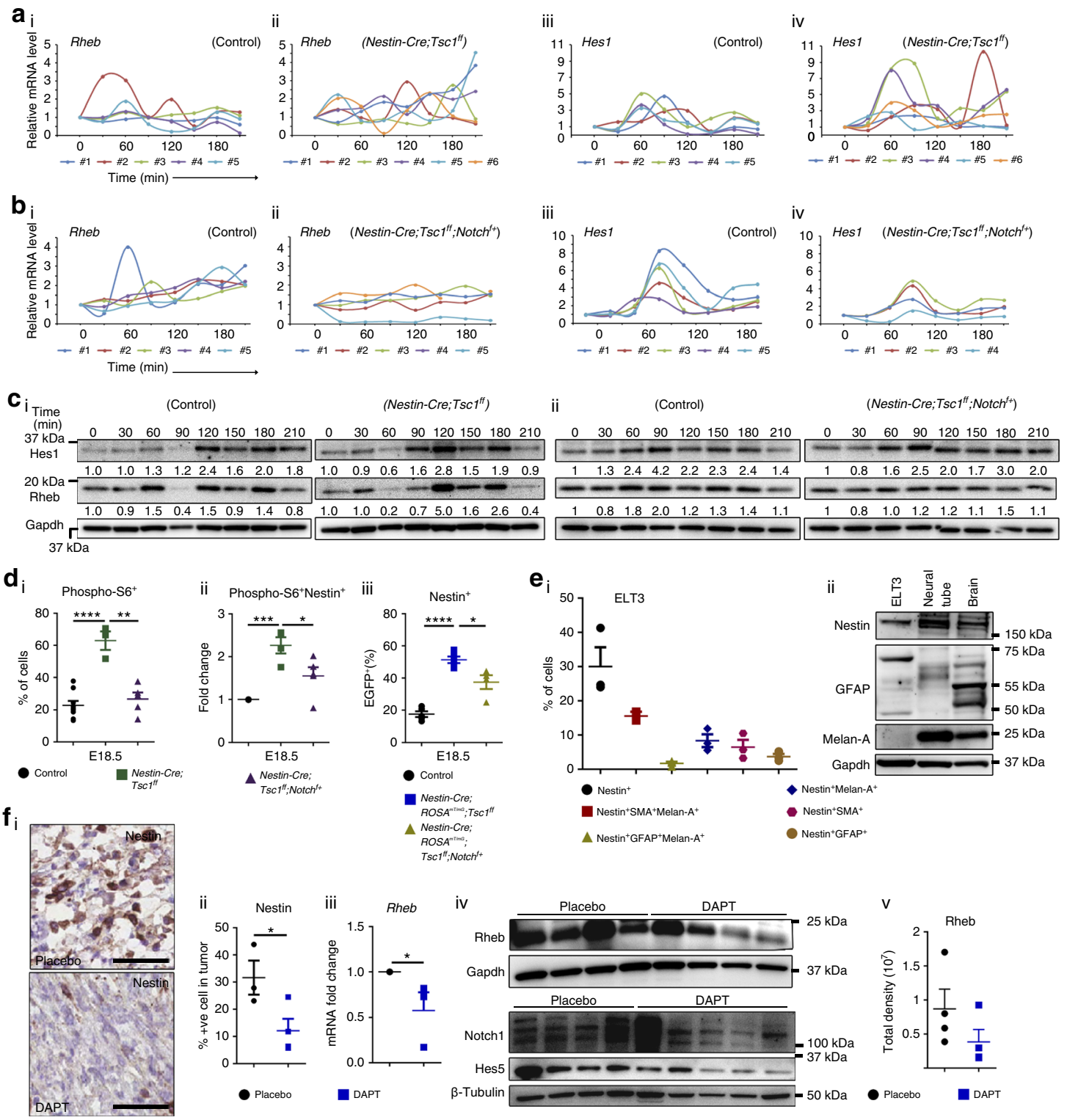

Fig. 6 Loss of Tsc1 leads to increased oscillation of Notch and Rheb. a, b q(RT)-PCR of (i, ii) Rheb and (iii, iv) Hes1 relative to Gapdh in NT cells from E15.5 littermate controls or a Nestin-Cre; Tsciff or b Nestin-Cre;Tsciff;Notch f+ embryos after release from synchronization. c The expression of Rheb and Hes1 in

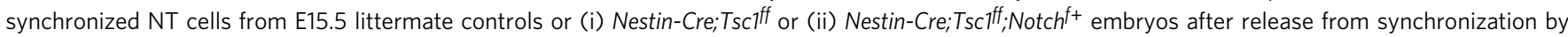
western immunoblotting. Numeric values represent densitometry analysis of the expression of Rheb or Hes1 relative to expression of Gapdh. $\mathbf{d}$ FACS of (i) phospho-S6 or (ii) phospho-S6 and nestin in NTs. d (i) percentage of phospho-S6 ${ }^{+}$cells in Nestin-Cre;Tsc ff and Nestin-Cre;Tsc ff $^{\text {;N Notch }}{ }^{f+}$ embryos relative to littermate control embryos ( $\mathrm{Tsc}^{++}{ }^{+} \mathrm{Notch}^{++}$), at E18.5. (ii) Fold changes in the percentage of phospho-S6 ${ }^{+}$nestin ${ }^{+}$NSCs in Nestin-Cre; Tsc $\mathrm{ff}^{\text {and }}$ aestin-Cre; Tsciff; Notch $^{f+}$ embryos relative to littermate control embryos $\left(\mathrm{TsCl}^{++} ; \mathrm{Notch}^{++}\right.$) at E18.5. (iii) The percentage of NSCs (EGFP ${ }^{+}$nestin ${ }^{+}$) within NTs from

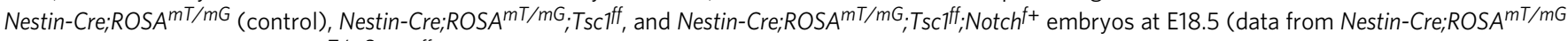
(control) and Nestin-Cre;ROSA ${ }^{\mathrm{mT} / \mathrm{mG}}$; Tsc ff mouse embryos are also used in Fig. $5 \mathrm{~d}(\mathrm{i}, \mathrm{ii})$ ). e (i) Percentages of ELT3 cells expressing nestin alone or co-expressing Melan-A, GFAP, and SMA by FACS. (ii) The expression of nestin, GFAP, and melan-A in ELT3 cells by western immunoblotting. $\mathbf{f}$ (i) Nestin expression in placebo- (top) and DAPT-treated (bottom panel) xenograft tumors. (ii) Quantification of results shown in $\mathbf{f}$ (i). (iii) q(RT)-PCR of Rheb relative to Gapdh. (iv) Rheb, Notch1 and Hes5 in placebo- $(n=6)$ and DAPT-treated $(n=5)$ xenograft tumors by western blot. (v) Quantification of Rheb expression shown in $\mathbf{f}$ (iv) by densitometry analysis after normalization to Gapdh. Data represent means \pm s.e.m.. Error bars are defined as means + s.e.m. ${ }^{\star} P \leq 0.05^{\star \star \star} P \leq 0.01,{ }^{\star \star \star} P \leq 0.001,{ }^{\star \star \star \star} P \leq 0.0001 t$-test. Data are representative of four, $\mathrm{n}_{\text {control }}=5(\mathbf{a}(\mathrm{i})), \mathrm{n}_{\text {Nestin-Cre; Tsc1ff }}=6\left(\mathbf{a}\right.$ (ii)), $\mathrm{n}_{\text {control }}=5(\mathbf{a}($ iii)), $\mathrm{n}_{\text {Nestin-Cre; Tsclff }}=6$ (a (iv)); three, $\mathrm{n}_{\text {control }}=5, \mathrm{n}_{\text {Nestin-Cre;Tsclff;Notchf+ }}=5(\mathbf{b}(\mathrm{i}-\mathrm{iii})), \mathrm{n}_{\text {Nestin-Cre;Tsclff;Notchf }}=4$ (b (iv)); two (c); three (d, e), $n=3(\mathbf{d}(\mathrm{i}, \mathrm{ii})$ ), $n=4(\mathbf{e}(\mathrm{i}), \mathbf{d}(\mathrm{iii})), n=3(\mathbf{f}(\mathrm{ii}, \mathrm{iii})), n=4(\mathbf{f}(\mathrm{v}))$ independent experiments

urinary pole progenitors have smaller cytoplasm compared to large tumor cells and tubules (Fig. 7h). Although the cells that give rise to tumors in Tsc1-null mice are expected to express nestin, only rare tumor cells preserved this expression, likely because of inactivity of the endogenous (mouse) nestin promoter upon differentiation of tumor cells. In summary, our results support the concept that the loss of Tsc1 blocks differentiation via the continuously active Rheb-Notch-Rheb loop. This loop activity is maintained by cyclic and continuous binding of Notch 1 to the activating NREs on the Rheb and Hes1 promoters. This mechanism facilitates increases in the expression and/or amplitudes of Rheb and Hes 1 oscillation (Fig. 7i). 
a

ii

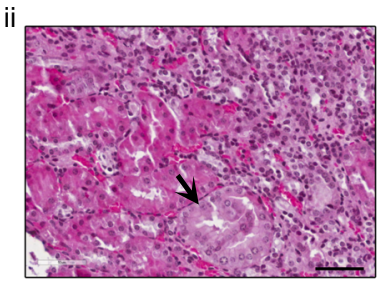

d

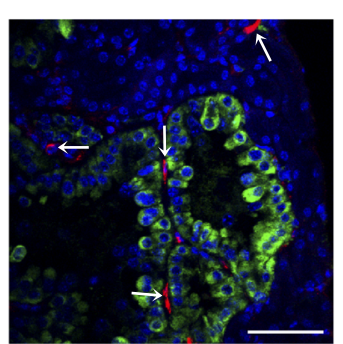

b
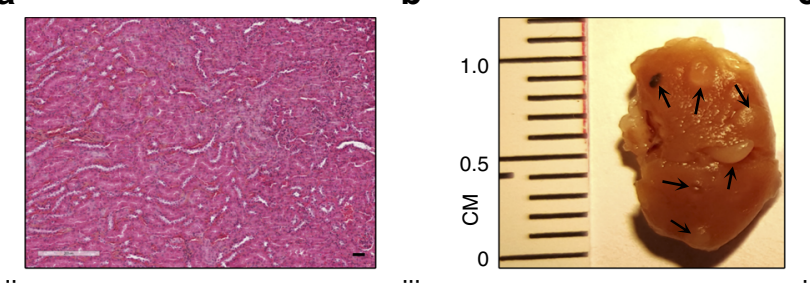

iii
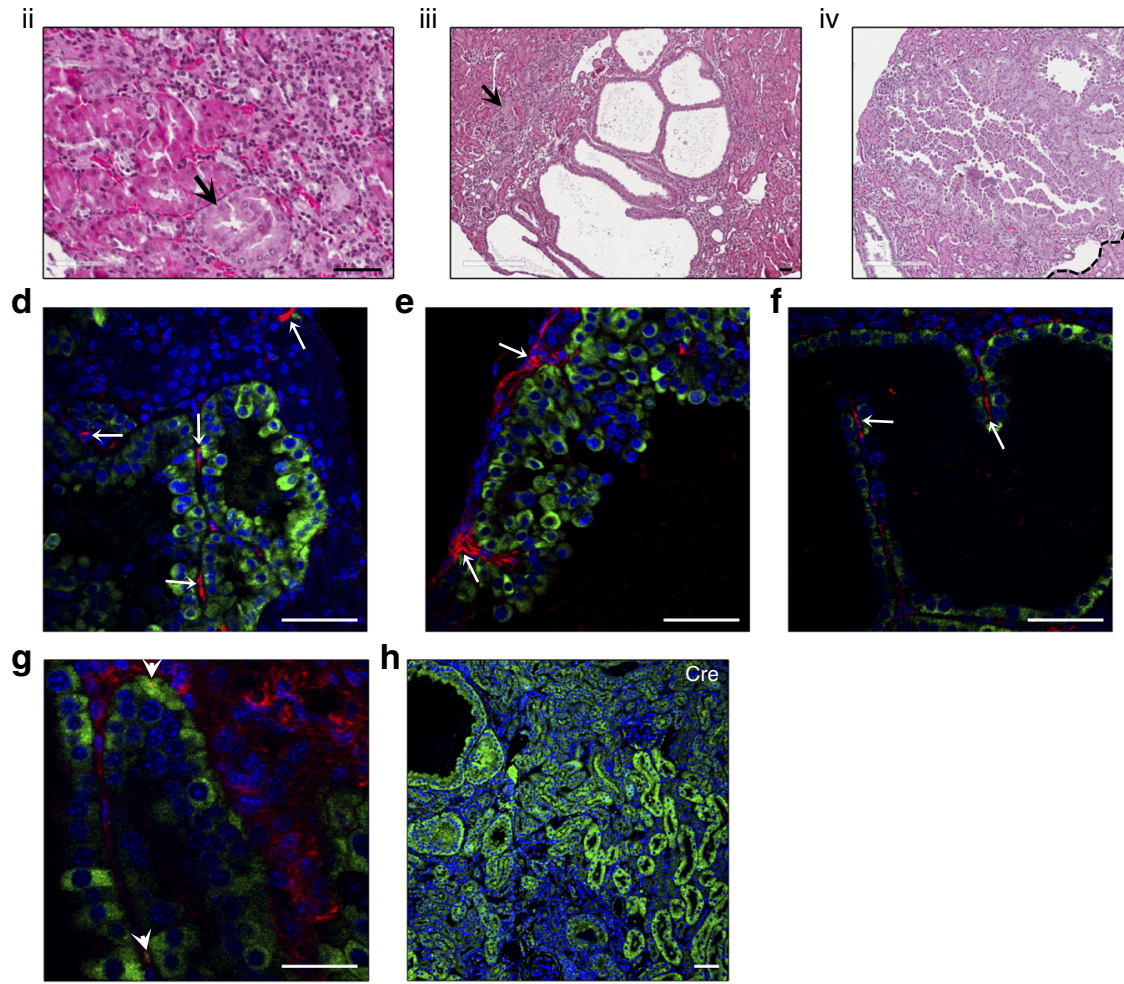

$\mathbf{c}_{\mathrm{i}}$

iv

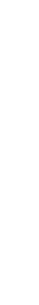

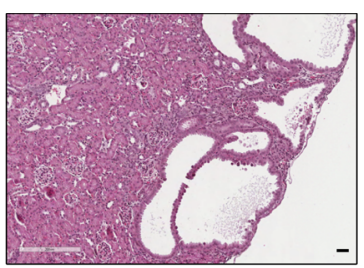
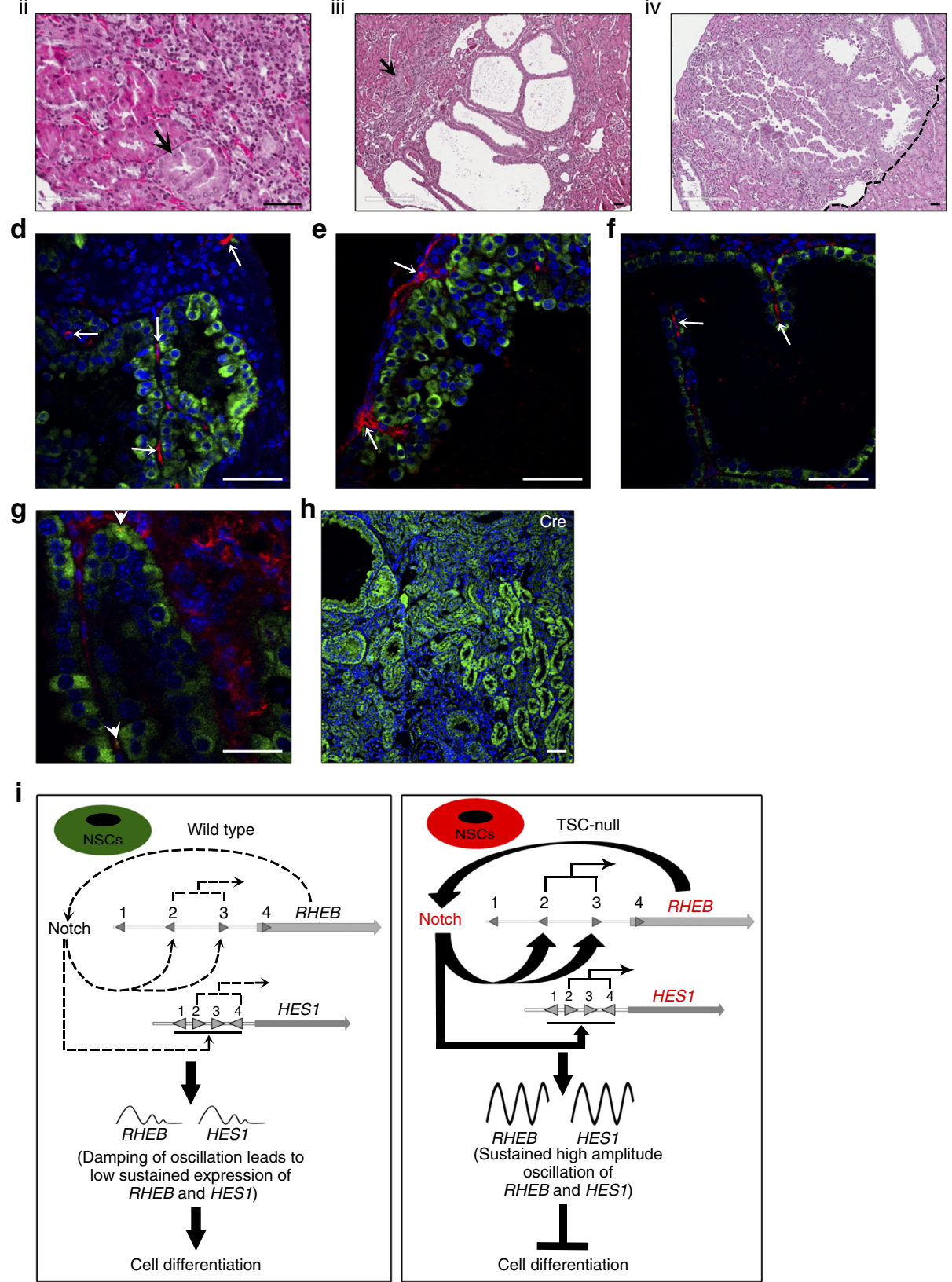

Fig. 7 Spontaneous renal tumorigenesis induced by cell-type-specific loss of Tsc1. a H\&Es of Tsc $T^{f / f}$ (control) kidney, $n=3$. b Kidney lesions in NestinTamCre; Tsc If/f; c H\&Es of Nestin-TamCre; Tsc fl/f kidneys, $n=3$ : (i) cysts; (ii) RIN (arrow); (iii) papillary carcinoma (micro-invasion-arrow); (iv) invasive papillary renal cell carcinoma (dashed line indicates border between RCC and normal kidney). Tamoxifen was injected intraperitoneally into 2-3-month-old mice at the dose of $120 \mathrm{mg} / \mathrm{kg} /$ day for two consecutive days. Mice were harvested at the age of 7 months. d-f Expression of nestin (red) in renal spindleshape tumor cells. Expression of phospho-S6 within renal lesions (green). $\mathbf{g}$ Co-expression of nestin and phospho-S6 in spindle-shape tumor cells (arrowheads). h Expression of Cre recombinase within renal lesions of Nestin-TamCre;Tsc $7^{f / f}$ mice. $\mathbf{i}$ The role of the Rheb-Notch-Rheb loop during NSC maintenance. The loss of Tsc1 blocks the differentiation of NSCs and progenitors via activation of the Rheb-Notch-Rheb loop, which leads to the amplified oscillation of HES1 and RHEB, controlled by the fluctuation in the binding of N1ICD to their activating NREs 


\section{Discussion}

We discovered a previously unreported binding of Notch1 to NREs in the promoter of Rheb that regulates Rheb transcription. This binding is a part of the novel regulatory mechanism, which we termed the Rheb-Notch-Rheb loop. We have focused only on NRE2 and NRE3, since the mutation of these sites resulted in significant difference in the activity of Rheb promoter. The NRE2 and NRE3 appear to be regulators for Notch-dependent transactivation of Rheb. We propose that reduction in activity of NRE2 and NRE3 on the Rheb promoter allows differentiation, since suppression of Rheb via NRE2 and NRE3 becomes apparent during experimentally induced neuronal differentiation. These findings provide insights into mechanisms of aberrant LAM and angiomyolipoma differentiation, since hyperactivation of this loop appears to maintain multipotency of angiomyolipoma cells. Conversely, reduced activity of this loop permits differentiation of angiomyolipoma cells along neuronal lineages. We recognize that the potential RBPJ binding site 1 and NRE2 sites are only 212 bps apart and it may be difficult to distinguish between loading of these by Notch1-ternary complex as a result of their proximity. However, we see consistent and stronger binding of Notch1 to NRE2 and NRE3, but not to the potential RBPJ binding site 1, suggesting that the latter is rather inactive. Our conclusion is supported by suppression of Notch1-ternary complex binding to NRE2 and NRE3 in N-medium and lack of such effect for potential RBPJ binding site 1 . The interaction between Notch1 and Rheb might also involve the regulation of Rheb at the protein level. Makovski et al. ${ }^{61}$ determined that Rheb protein is stabile for at least $26 \mathrm{~h}$. We observed the significant reduction of Rheb at the protein level within $90 \mathrm{~min}$ from release from serum synchronization (Figs. 2b and $6 \mathrm{c}$ ). Therefore, it is likely that Notch1 also regulates Rheb protein degradation to achieve its oscillatory expression, similar to the regulation of $\beta$-catenin protein degradation in stem and progenitor cells ${ }^{62}$. The angiomyolipoma cell line (CRL4004) has only one inactivated and one normal allele of TSC2, and therefore some of data obtained with this cell line should be interpreted with caution. However, both angiomyolipoma cell lines 621-101 and CRL4004 used in this work have multiple common features such as mTORC1 hyperactivation, differentiation abnormalities, binding of Notch 1 to the RHEB and HES1 promoters, and oscillation of RHEB and HES1 mRNAs and proteins. Therefore, we consider CRL4004 cell line as an appropriate tool to study angiomyolipoma in addition to wellestablished 621-101 cells.

The loss of Tsc1 in mouse embryo NTs leads to the accumulation of NSCs, inhibition of differentiation, and increased oscillation of Hes1 and Rheb expression, consistent with our findings in angiomyolipoma cells. This is also consistent with the important function of Notch1 in maintaining high level of Hes1, which retains NSCs in an undifferentiated state. Therefore, the Rheb-Notch-Rheb loop appears to regulate NSC differentiation during embryonic development (Fig. 7i). In addition, we provide evidence for the role of this loop in maintenance of Tsc-null multipotent tumor cells during TSC tumorigenesis.

In summary, we have provided direct and indirect evidence for the significant role of the Rheb-Notch-Rheb loop in maintaining multipotent properties of Tsc-null cells and TSC tumorigenesis, although certain aspects of the proposed model remain to be confirmed. In addition, our data suggest the potential origin for TSC-associated RCC from renal progenitors localized in the Bowman's capsule urinary pole, although more thorough investigation is required to fully support this notion. These data are clinically relevant, since renal cysts are found in 50\% of TSC patient and $2-4 \%$ of these patients develop TSC-associated papillary $\mathrm{RCC}^{60}$. Although Tsc1/2 heterozygosity has been reported to be associated with RCC and cysts in mice ${ }^{63,64}$, our data point to a role of nestin-expressing kidney cells in earlyonset RCC.

Our data indicate that Notch1 transactivates Rheb. Rheb mediates the activation of $\mathrm{mTOR}^{20}$, and mTOR is activated in Notch-dependent malignancies ${ }^{65}$, and therefore this study suggests a potential role for the Rheb-Notch-Rheb loop in mTOR activation in non-TSC malignancies without identified TSC1/2 mutations. Therapeutic targeting of this loop might offer a new strategy for patients with Notch/Rheb/mTORC1-dependent tumors.

\section{Methods}

Human and animal studies. Human samples were from the National Disease Research Interchange (NDRI) and the Center for LAM Research at Brigham and Women's Hospital with obtained informed consent from all human participants under Institutional Review Board approval.

The following mouse strains were used: B6.Cg-Tg(Nes-cre)1Kln/J, C57BL/6-Tg (Nes-cre/Esr1)1Kuan/J, FVB-Tg(GFAP-cre)25Mes/J, Tsc1tm1Djk/J, B6.129×1Notch $1^{\text {tm } 2 R k o} /$ GridJ, and Gt(ROSA)26Sortm4(ACTB-tdTomato,EGFP)Luo/J (The Jackson Laboratory). Animals, females and males, were on C57BL/6J, FVB/N or mixed C57BL/6J, BALB/cJ, 129/SvJae background. For the inducible promoter tamoxifen dissolved in corn oil $(40 \mathrm{mg} / \mathrm{ml})$ was administered intraperitoneally (i.p.; $120 \mathrm{mg} / \mathrm{kg} /$ day) for 2 days. Embryos with similar genotype were used in randomized cohorts. Xenograft tumor volume: ELT3 cells were inoculated into the posterior back region of 6-week-old immunodeficient SCID mice (Charles River Laboratories). Tumor length, width, and depth were monitored with a Vernier caliper. When tumors reached $125 \mathrm{~mm}^{3}$, mice were randomly assigned to DAPT (Calbiochem) at the dose of $10 \mathrm{mg} / \mathrm{kg} /$ day or vehicle control via i.p. injection for 3 consecutive days, followed by 4 -day interval up to 2 weeks ${ }^{17}$. Animal experiments were approved by the Texas Tech University Health Sciences Center Institutional Animal Care and Use Committee according to the National Institutes of Health (NIH) guidelines.

Cells. Angiomyolipoma-derived cells: 621-101 with inactivation of both TSC2alleles $^{28}$ (from Dr. Elizabeth Henske), CRL4004 (ATCC) with a 5 bp deletion in TSC2 exon $33^{30}$ from a sporadic angiomyolipoma ${ }^{29}$ were cultured in DMEM (high glucose) media (Corning) supplemented with $10 \%$ fetal bovine serum (FBS) or heat-inactivated FBS, penicillin/streptomycin, and glutamine ${ }^{17} .621-101$ and CRL4004 cells were plated in N-medium immediately after trypsynization. Nmedium was prepared as follows: DMEM:F12 (Sigma-Aldrich), 15\% chicken embryo extract (U.S. Biological Inc. or VWR), N2 and B27 supplements (Life Technology), $20 \mathrm{ng} / \mathrm{ml}$ recombinant human fibroblast growth factor-basic (Prospec), $20 \mathrm{ng} / \mathrm{ml}$ recombinant human insulin-like growth factor 1 , and $20 \mathrm{ng} / \mathrm{ml}$ human recombinant epidermal growth factor (R\&D System $)^{44}$.

Fresh aliquots of HeLa and 293T cells were purchased from ATCC and cells were cultured in DMEM (high glucose) supplemented with 10\% FBS, penicillin/ streptomycin, and glutamine.

SK-Mel2, SK-Mel5, and Malme (all from ATCC) were maintain in RPMI supplemented with $10 \%$ heat-inactivated FBS, penicillin/streptomycin, and glutamine. ELT3 cells (from Dr. Elizabeth Henske) were maintained in IIA complete medium (50\% DMEM:F12, $1.2 \mathrm{~g} / \mathrm{L}$ NaHCO3, $1.6 \mu \mathrm{M}$ FeSO4, $50 \mathrm{nM}$ sodium selenite, $25 \mu \mathrm{g} / \mathrm{ml}$ insulin, $200 \mathrm{nM}$ hydrocortisone, $10 \mu \mathrm{g} / \mathrm{ml}$ transferrin, 1 $\mathrm{nM}$ triiodothyronine, $10 \mu \mathrm{g} / \mathrm{ml}$ vasopressin, $10 \mathrm{nM}$ cholesterol, $10 \mathrm{ng} / \mathrm{ml}$ epidermal growth factor, $15 \% \mathrm{FBS}$, and penicillin/streptomycin ${ }^{59}$ ). Cells were treated with 1 nM rapamycin (Biomol) or dimethyl sulfoxide. Cells identity was verified by the STR-DNA profiling (Genetica). All cell lines were routinely tested for mycoplasma contamination (InvivoGen). Stable lines were generated by infection with lentiviruses packaged with psPAX2 and pMD2.G vectors containing control, Rheb shRNA (Addgene) ${ }^{46}$, or Notch shRNA (Sigma) and selected with $8 \mu \mathrm{g} / \mathrm{ml}$ puromycin 66

Mutational analysis of CRL4004. The comprehensive TSC1/TSC2 genetic analysis was performed by massively parallel sequencing using a customized gene bait set, yielding $500 \times$ coverage of the entire genomic extent of TSC1 and TSC2. This analysis confirmed the known frameshift TSC2 mutation c.4081_4085delCGAGT/ p.V1362Lfs ${ }^{*}$ with allele frequency $48 \%$ (hg19) (previously reported by Lim et al. ${ }^{30}$ ) in the CRL4004 cell line. Allele frequency single-nucleotide polymorphism analysis in TSC1/TSC2 genes showed no hint for loss of heterozygosity (LOH) in CRL4004 cell line (skewed allele frequency $\mathrm{AF}<0.4$ or $\mathrm{AF}>0.6$ ). This highly sensitive analysis strongly suggests that the CRL4004 cell line has one inactivated and one normal allele of TSC2.

Western blotting. Cells were lysed using RIPA, Tris-Triton buffer (for an intermediate filament, microtubules, or cytoskeletal proteins) (10 mM Tris, $\mathrm{pH} 7.4,100$ $\mathrm{mM} \mathrm{NaCl}, 1 \mathrm{mM}$ EDTA, $1 \%$ Triton X-100, 10\% glycerol, $0.1 \%$ SDS, $0.5 \%$ deoxycholate) or nuclear extraction buffer (20 mM HEPES, pH 7.9, with $1.5 \mathrm{mM} \mathrm{MgCl}$ $0.42 \mathrm{M} \mathrm{NaCl}, 0.2 \mathrm{mM}$ EDTA, and $25 \%(\mathrm{v} / \mathrm{v})$ glycerol). Then, $5 \mu \mathrm{l}$ of $0.1 \mathrm{M}$ 
dithiothreitol solution and protease and phosphatase inhibitor cocktails were added to $500 \mu \mathrm{l}$ of $1 \times$ lysis buffer. Lysates were subjected to electrophoresis (Invitrogen).

Immunoassays. The following antibodies were used: Rheb (Cell Signaling \#13879, $1: 1000$ ), NSE (Abcam \#ab16808, 1:1000), $\beta$-3-tubulin (Abcam \#ab78078, 1:200 (IF), 1:5000 (WB)), nestin (Abcam \#ab6142, 1:100 (IF)), peripherin (Abcam \# ab4666, 1:1000), GFAP (Sigma-Aldrich \# G9269, 1:200 (immunohistochemistry (IHC)), 1:1000 or 1:5000 (WB)), $\beta$-actin (Sigma-Aldrich \#A5316, 1:2000), $\beta$-tubulin (Sigma-Aldrich \# T8328, 1:5000) c-myc (Santa Cruz Biotechnology \#sc-40, 1:500), Notch1 (C-20) (Santa Cruz Biotechnology \#sc-6014-R, 1:500), Hes5 (Santa Cruz Biotechnology \#13859, 1:500); NSE (BioGenex, \# MU055-UC, 1:50 (IHC)); nestin (Novus Biologicals \# NBP1-02419 1:1000); cleaved Notch1 (Val1744) (Cell Signaling \#4147, 1:1000), hamartin (Cell Signaling \#4906, 1:1000), phospho-S6 (Cell Signaling \#2211, 1:1000 (WB), 1:400 (IF)), hes1 (Cell Signaling \#11988, 1:1000 (WB)), hes1 (Genetex \#GTX62458, 1:200 (IF)), phospho-S6K (T389) (Cell Signaling \#9202, 9206, 1:1000) and Gapdh (Cell Signaling \#5174P, 1:3000); phosphoS6-PacificBlue (Cell Signaling \#8520, 1:320), nestin-PE/AF647 (BD Biosciences \#561230, 1:100), GFAP-PE/AF647 (BD Biosciences \#561470, 1:40), $\beta$-3-tubulinFITC (Sigma-Aldrich \#SAB4700545, 1:100), melan-A (Thermo Scientific \#MA5 15237, 1:500), SMA-PerCP (R\&D Systems \#IC1420C, 1:10), Ki-67-PE/Cy7 (BD Biosciences \#561283, 1:80); Cre recombinase (EMD Millipore \# MAB3120, 1:500 (IF)). Secondary steps for IHC were performed using detection kit (Invitrogen). Images were captured with Nikon TE2000 microscope. FACS events were acquired using LSRFortessa (BD Bioscience).

IHC quantification using Aperio. Aperio software system removes all the background threshold prior to analysis, and therefore the area of co-localization is free of background error. Aperio digital pathology systems uses algorithm-based software analysis to locate and quantitate the staining. The co-localization algorithm separates the stains based on the RGB (red, green and blue) values into three channels corresponding to the colors used, allowing quantifying the area and intensities of each stain separately and the co-localized markers. After running the algorithm analysis, the software generates a 'Markup image' with an arbitrary color for each stain and for areas where stains co-localize. The negative areas with only hematoxylin are marked in Blue, Red and Green for individual stained areas and Yellow in the areas where the stains superimpose. The yellow color is arbitrary choice by the Aperio software to indicate areas of co-localization and it does not reflect the color resulting from mixing two different colors as seen during immunofluorescence detection.

\section{Transfection and reporter gene assays. Human MAML1 and N1ICD were} cloned into pCS2 containing an N-terminal 6X-MycTag using Gateway technology and standard PCR. Rheb luciferase and control promoter constructs were purchased from Lightswitch Genomics (catalog number S717541). Hela cells were seeded at 20,000 cells per well in 96-well plate format. The next day, the media were changed to Opti-MEM I and cells were transiently transfected with $75 \mathrm{ng}$ of the Rheb-Promoter construct, $10 \mathrm{ng}$ of pGL3-Control, $50 \mathrm{ng}$ of N1ICD, and $50 \mathrm{ng}$ of MAML1 and $35 \mathrm{ng}$ of pCS2 using Lipofectamie 2000 transfection reagent (Invitrogen). Cells were harvested $48 \mathrm{~h}$ later in $50 \mu \mathrm{l}$ of $1 \times$ Passive Lysis Buffer and the levels of luciferase were measured with the Dual Luciferase Assay System from Promega on a Modulus II Luminometer with $10 \mathrm{~s}$ integration time per luciferase assay and $2 \mathrm{~s}$ delay before taking the reading. The readings were obtained in Microsoft Excel software. The measurement readings were given as relative light units, and analyzed as a ratio of the Renilla luciferase measurements to the control Firefly luciferase measurements.

Site-directed mutagenesis. The Rheb-NRE mutants were generated as previously described $^{43}$ using QuikChange (Stratagene) and the following primers: NRE1MUT-S-5'CAGACTCCATGCTTGCGACAGTCCTTCGGCC-3'/NRE1-MUT-AS 5'-GGCCGAAGGACTGTCGCAAGCATGGAGTCTG-3', NRE2-MUT-S-5'CAA TCACCGCACCTGCGACCTATTGCCCCGC-3'/NRE2-MUT-AS-5'-GCGGGG CAATAGGTCGCAGGTGCGGTGATTG-3', NRE3-MUT-S-5'-CCTTGTTTC CCCCAATCGCAGATGGAGTTTCCG-3'/NRE3-MUT-AS-5'-CGGAAACTCCATCTGCGATTGGGGGAAACAAGG-3', NRE4-MUT-S-5'-AAAGCGGCGGAAGAAGGTCGCAGGGTCATGAC-3'/NRE4-MUT-AS-5'- GTCATGACC CTGCGACCTTCTTCCGCCGCTTT-3'. For each mutant, Sanger sequencing (Genewiz) verified the integrity of the whole promoter region and base pair changes.

Genome editing with CRISPR Cas9. The single-guide RNAs (sgRNAs) targeting genomic regions of interest were designed using CRISPR Design Tool (http:// portals.broadinstitute.org/gpp/public/) and the guide sequences are as follows: NRE3 G2'-S-5'-CACCGAGCTGTCCAATCGGCGCTCG-3'/NRE3 G2'-AS-5'-AA ACCGAGCGCCGATTGGACAGCTC-3'/NRE3-G3'-S-5'-CACCGGTGTATT TTTAGCTCCCGGG-3'/NRE3-G3' ${ }^{\prime}$-AS-5' ${ }^{\prime}$-AAACCCCGGGAGCTAAAAATACACC- $3^{\prime}$. The sgRNA oligos were subcloned into pSpCas9(BB)-2A-GFP (PX458) Addgene vector and transiently transfected into CRL4004 cells. At $48 \mathrm{~h}$ post transfection, cells were sorted using FACS Aria (BD) and plated to 96-wells plate at a density of 0.8 cell per well. Cell grown up to $90 \%$ confluency underwent subculture for maintenance, genomic purification and PCR, and western immunoblotting analyses. For PCR amplification of NRE3 region, NRE3-S-5'-CGGT AGCAGCGAGGTGTATT- ${ }^{\prime}$ and NRE4-AS-5'-GTCGGGGCGACGTTTTACTT$3^{\prime}$ primers were used and clones were screened. Amplicons of $272 \mathrm{bp}$ indicated intact DNA. Amplicons of $165 \mathrm{bp}$ indicated clones with prospective focal deletion of NRE3 region and those were subjected for sequencing.

Neural tube dissociation was performed using papain according to the manufacturer (Worthington)

Real time quantitative reverse transcription. The $\mathrm{q}(\mathrm{RT})-\mathrm{PCR}$ was performed using High Capacity cDNA Synthesis Kit, Fast SybrGreen, StepOnePlus Applied Biosystems and the following primers: SOX10-F-5'-ATGAACGCCTTCATGG TGTGGG-3'/R-5'-CGCTTGTCACTTTCGTTCAGCAG-3'; NGN1-F-5'-CCCCT AGTCAGCAGGCAATA-3'/R-5'-CCTAACAAGCGGCTCAGGTA-3'; S100A1-F5'-TGGACTTCCAGGAGTATGTGG-3'/R-5'-TGCTCAACTGTTCTCCCAGA-3'; DCT-F-5'-CCTAGGGTGCTCATGCCTTA-3'/R-5'-CAACTCAAGAAGGAACA GTGAGG-3'; c-KIT-F-5'-GCAAATACACGTGCACCAAC-3'/R-5'-GCACCCCTT GAGGGAATAAT-3'; ID1-F-5'CCAACGCGCCTCGCCGGATC-3'/R-5'-CTCCT CGCCAGTGCCTCAG-3'; ID3-F-5'-CAGCTTAGCCAGGTGGAAATCC-3'/R-5' GTCGTTGGAGATGACAAGTTCCG-3'; SOX9-F-5'-AGGAAGCTCGCGGACCAGTAC-3'/R-5'-GGTGGTCCTTCTTGTGCTGCAC-3'; NOTCH1 and RHEB (human and mouse, both from Qiagen or Applied Biosystem/TaqMan); Rheb (rat)F-5'-CTGACCAGGCTACCAAGATG-3'/R-5'-CAATGAGGACTTTCCCACAGA3'; HES1-F-5'-GGAAATGACAGTGAAGCACCTCC/R-5'- GAAGCGGGTCAC CTCGTTCATG-3'; Hes1-(from Applied Biosystem/TaqMan)'; Hes1-(rat)-F-5'CAACACGACACCGGACAAAC-3'/ R-5'-GGAATGCCGGGAGCTATCTT - $3^{\prime}$; GAPDH- F-5'-ACTGACACGTTGGCAGTGG-3'/ R-5'-GGCTCTCCAGAACATCATCC-3'; Gapdh-(mouse)-(from Applied Biosystem/TaqMan); Gapdh-(rat)F-5'-GACATGCCGCCTGGAGAAAC-3'/R-5'-AGCCCAGGATGCCCTTT AGT-3'; 18S-F-5'-GTCTGTGATGCCCTTAGATG-3'/R-5'-AGCTTATGACC CGCACTTAC- $3^{\prime}$.

Relative expression was calculated using the $2-\Delta \Delta \mathrm{Ct}$ method and RT2 profiler PCR Array Data Analysis (SAB Biosciences) and normalized to GAPDH.

Chromatin immunoprecipitation quantitative PCR. ChIP-qPCR was performed using EZ-ChIP (Millipore) or SimpleChIP Enzymatic Chromatin IP kit (Cell Signaling). Immunoprecipitation was performed using $\alpha$-Notch1 (Abcam) or IgG control. qPCR for Rheb-NREs was performed using primers: NRE-1-S-5'-CG AGGCTCGCCCTGTTTTTA-3'/NRE-1-AS-5'-CTTGAGCTGCGTTAGCGTTC3', NRE2-S-5'-ACCCAGACACGAACGCTAAC-3'/NRE2-AS-5'-GAGAGACAA ACGGTGGCTCC-3'; NRE-3-S-5'-CGGTAGCAGCGAGGTGTATT-3'/NRE-3AS-5'-GATTGGACAGCTCCTGCACA-3'. For Notch binding to Hes1 promoter the following primers were used: HES1-S-5'-GCGTGTCTCCTCCTCCCATT- $3^{\prime}$ / HES1-AS-5'-CCTGGCGGCCTCTATATATA- $3^{\prime 67}$.

RNA sequencing analysis. The preprocessed RNA-Seq data were imported into the Partek Flow (Partek, CA). The genome versions of Homo sapiens hg19 and STAR aligner were used for alignment and alignment algorithm. Quantification and normalization were performed with an expectation maximization algorithm. Genes displaying differential expression between normal kidney from patient with sporadic angiomyolipoma, angiomyolipoma tumor, and two angiomyolipomaderived cell lines (621-101 and CRL4004) were detected using poisson model, assuming that the variance is equal to the mean. The $P$-value is obtained using Wald. $P$-values were adjusted to account for multiple testing using the false discovery rate (FDR) method of Storey and Tibshirani. Only genes that were significantly $($ FDR $<0.05)$ modulated in angiomyolipoma tumor and two angiomyolipoma-derived cell lines (621-101 and CRL4004) compared with norma kidney were considered for further analysis.

To assess biological relationships among genes, we used the Ingenuity Pathway Analysis software (QIAGEN Inc.). Network analyses, functional analyses, and canonical pathway analyses were generated.

Statistical analysis. Kolmogorov-Smirnov, unpaired $t$-tests, one-way or two-way analysis of variance (ANOVA), non-parametric Mann-Whitney tests, or the regression analysis of longitudinal data (repeated measures) were used. Standard error of means (s.e.m.) was estimated. The variance was similar between groups that were compared. For normalized $\mathrm{q}(\mathrm{RT})$-PCR, fold changes, FACS, and densitometry analyses one-sample $t$-tests were used. Outliers were identified using Grubb's test at $\alpha=0.05$. Analyses were done using GraphPad Prism 6 and Statistica.

Based on power calculation using the non-central $t$-distribution for a two-sided $t$-test, we have estimated that analysis of 10 mice per cohort allow us to achieve an actual power of $75 \%$ power to distinguish an effect, defined as the difference in means relative to the standard deviation, of 1.25 between two treatments at the $5 \%$ level of significance. However, the preliminary studies have demonstrated that the inclusion of 5 subjects per cohort is sufficient. For animal studies analyses were performed by researcher blinded to sample identity. 
Data availability. The authors declare that data supporting the findings of this study are available within the paper and its supplementary information files. All additional relevant data are available from the authors.

Received: 23 May 2016 Accepted: 20 October 2017

Published online: 29 November 2017

\section{References}

1. Meacham, C. E. \& Morrison, S. J. Tumour heterogeneity and cancer cell plasticity. Nature 501, 328-337 (2013).

2. Lasorella, A., Benezra, R. \& Iavarone, A. The ID proteins: master regulators of cancer stem cells and tumour aggressiveness. Nat .Rev. Cancer 14, 77-91 (2014).

3. Bedard, P. L., Hansen, A. R., Ratain, M. J. \& Siu, L. L. Tumour heterogeneity in the clinic. Nature 501, 355-364 (2013).

4. Wong, M. Mechanisms of epileptogenesis in tuberous sclerosis complex and related malformations of cortical development with abnormal glioneuronal proliferation. Epilepsia 49, 8-21 (2008).

5. Chan, J. K. et al. Lymphangiomyomatosis and angiomyolipoma: closely related entities characterized by hamartomatous proliferation of HMB-45-positive smooth muscle. Histopathology 22, 445-455 (1993).

6. Karbowniczek, M., Yu, J. \& Henske, E. P. Renal angiomyolipomas from patients with sporadic lymphangiomyomatosis contain both neoplastic and nonneoplastic vascular structures. Am. J. Pathol. 162, 491-500 (2003).

7. Morrison, S. J. et al. Transient Notch activation initiates an irreversible switch from neurogenesis to gliogenesis by neural crest stem cells. Cell 101, 499-510 (2000).

8. Artavanis-Tsakonas, S., Rand, M. D. \& Lake, R. J. Notch signaling: cell fate control and signal integration in development. Science 284, 770-776 (1999).

9. Mead, T. J. \& Yutzey, K. E. Notch signaling and the developing skeleton. $A d v$. Exp. Med. Biol. 727, 114-130 (2012).

10. Mead, T. J. \& Yutzey, K. E. Notch pathway regulation of neural crest cell development in vivo. Dev. Dyn. 241, 376-389 (2012).

11. Bao, Z. Z. \& Cepko, C. L. The expression and function of Notch pathway genes in the developing rat eye. J. Neurosci. 17, 1425-1434 (1997)

12. Radtke, F., Schweisguth, F. \& Pear, W. The Notch 'gospel'. EMBO Rep. 6, 1120-1125 (2005)

13. Weinmaster, G., Roberts, V. J. \& Lemke, G. A homolog of Drosophila Notch expressed during mammalian development. Development 113, 199-205 (1991).

14. Weinmaster, G. \& Kopan, R. A garden of Notch-ly delights. Development 133, 3277-3282 (2006)

15. Leong, K. G. \& Gao, W. Q. The Notch pathway in prostate development and cancer. Differentiation 76, 699-716 (2008).

16. Kerbel, R. S. Tumor angiogenesis. N .Engl. J. Med. 358, 2039-2049 (2008)

17. Karbowniczek, M. et al. The evolutionarily conserved TSC/Rheb pathway activates Notch in tuberous sclerosis complex and Drosophila external sensory organ development. J. Clin. Invest. 120, 93-102 (2010).

18. Ma, J. et al. Mammalian target of rapamycin regulates murine and human cell differentiation through STAT3/p63/Jagged/Notch cascade. J. Clin. Invest. 120, 103-114 (2010).

19 Shimojo, H., Ohtsuka, T. \& Kageyama, R. Oscillations in notch signaling regulate maintenance of neural progenitors. Neuron 58, 52-64 (2008).

20 Zhang, Y. et al. Rheb is a direct target of the tuberous sclerosis tumour suppressor proteins. Nat. Cell Biol. 5, 578-581 (2003).

21 Delaney, S. P., Julian, L. M. \& Stanford, W. L. The neural crest lineage as a driver of disease heterogeneity in tuberous sclerosis complex and lymphangioleiomyomatosis. Front. Cell Dev. Biol. 2, 69 (2014).

22 Locher, H., Frijns, J. H., Huisman, M. A. \& de Sousa Lopes, S. M. TUBB3: neuronal marker or melanocyte mimic?. Cell Transplant. 23, 1471-1473 (2014).

23 Lendahl, U., Zimmerman, L. B. \& McKay, R. D. CNS stem cells express a new class of intermediate filament protein. Cell 60, 585-595 (1990).

24 Neradil, J. \& Veselska, R. Nestin as a marker of cancer stem cells. Cancer Sci. 106, 803-811 (2015)

25 Thompson, M. A. \& Ziff, E. B. Structure of the gene encoding peripherin, an NGF-regulated neuronal-specific type III intermediate filament protein. Neuron 2, 1043-1053 (1989).

26 Wislet-Gendebien, S. et al. Plasticity of cultured mesenchymal stem cells: switch from nestin-positive to excitable neuron-like phenotype. Stem Cells 23, 392-402 (2005).

27 McDermott, K. W., Barry, D. S. \& McMahon, S. S. Role of radial glia in cytogenesis, patterning and boundary formation in the developing spinal cord. $J$. Anat. 207, 241-250 (2005)

$28 \mathrm{Yu}$, J., Astrinidis, A., Howard, S. \& Henske, E. P. Estradiol and tamoxifen stimulate LAM-associated angiomyolipoma cell growth and activate both genomic and nongenomic signaling pathways. Am. J. Physiol. Lung Cell. Mol. Physiol. 286, L694-L700 (2004).

29 Arbiser, J. L. et al. The generation and characterization of a cell line derived from a sporadic renal angiomyolipoma: use of telomerase to obtain stable populations of cells from benign neoplasms. Am. J. Pathol. 159, 483-491 (2001)

$30 \mathrm{Lim}, \mathrm{S}$. D. et al. Expression of the neural stem cell markers NG2 and L1 in human angiomyolipoma: are angiomyolipomas neoplasms of stem cells? $\mathrm{Mol}$. Med. 13, 160-165 (2007).

31 McLaren, F. H., Svendsen, C. N., Van der Meide, P. \& Joly, E. Analysis of neural stem cells by flow cytometry: cellular differentiation modifies patterns of MHC expression. J. Neuroimmunol. 112, 35-46 (2001).

32 Zhang, J. \& Jiao, J. Molecular biomarkers for embryonic and adult neural stem cell and neurogenesis. Biomed. Res. Int. 2015, 727542 (2015).

33 Kim, J., Lo, L., Dormand, E. \& Anderson, D. J. SOX10 maintains multipotency and inhibits neuronal differentiation of neural crest stem cells. Neuron 38, 17-31 (2003)

34 Sommer, L., Ma, Q. \& Anderson, D. J. neurogenins, a novel family of atonalrelated bHLH transcription factors, are putative mammalian neuronal determination genes that reveal progenitor cell heterogeneity in the developing CNS and PNS. Mol. Cell. Neurosci. 8, 221-241 (1996).

35 Woodhoo, A., Dean, C. H., Droggiti, A., Mirsky, R. \& Jessen, K. R. The trunk neural crest and its early glial derivatives: a study of survival responses, developmental schedules and autocrine mechanisms. Mol. Cell. Neurosci. 25, 30-41 (2004).

36 Falcon, S., . \& Gentleman, R. Using GOstats to test gene lists for GO term association. Bioinformatics 23, 257-258 (2007).

37 Rodgers, J. T. et al. mTORC1 controls the adaptive transition of quiescent stem cells from G0 to G(Alert). Nature 510, 393-396 (2014).

38 Tyson, J. J., Chen, K. \& Novak, B. Network dynamics and cell physiology. Nat. Rev. Mol. Cell Biol. 2, 908-916 (2001).

39 Pourquie, O. The segmentation clock: converting embryonic time into spatial pattern. Science 301, 328-330 (2003).

40 Hirata, $\mathrm{H}$. et al. Oscillatory expression of the bHLH factor Hes1 regulated by a negative feedback loop. Science 298, 840-843 (2002).

41 Yoshiura, S. et al. Ultradian oscillations of Stat, Smad, and Hes1 expression in response to serum. Proc. Natl. Acad. Sci. USA 104, 11292-11297 (2007).

42 Fryer, C. J., Lamar, E., Turbachova, I., Kintner, C. \& Jones, K. A. Mastermind mediates chromatin-specific transcription and turnover of the Notch enhancer complex. Genes Dev. 16, 1397-1411 (2002).

43 Kovall, R. A. \& Hendrickson, W. A. Crystal structure of the nuclear effector of Notch signaling, CSL, bound to DNA. EMBO J. 23, 3441-3451 (2004).

44 Pardal, R., Ortega-Saenz, P., Duran, R. \& Lopez-Barneo, J. Glia-like stem cells sustain physiologic neurogenesis in the adult mammalian carotid body. Cell 131, 364-377 (2007).

45 Prieto, V. G., McNutt, N. S., Lugo, J. \& Reed, J. A. The intermediate filament peripherin is expressed in cutaneous melanocytic lesions. J. Cutan. Pathol. 24, 145-150 (1997).

46 Sancak, Y. et al. The Rag GTPases bind raptor and mediate amino acid signaling to mTORC1. Science 320, 1496-1501 (2008).

47 Karbowniczek, M., Robertson, G. P. \& Henske, E. P. Rheb inhibits C-raf activity and B-raf/C-raf heterodimerization. J. Biol. Chem. 281, 25447-25456 (2006)

48 Nichane, M. et al. Hairy2-Id3 interactions play an essential role in Xenopus neural crest progenitor specification. Dev. Biol. 322, 355-367 (2008).

49 Scott, C. E. et al. SOX9 induces and maintains neural stem cells. Nat. Neurosci. 13, 1181-1189 (2010).

50 Lyden, D. et al. Id1 and Id 3 are required for neurogenesis, angiogenesis and vascularization of tumour xenografts. Nature 401, 670-677 (1999).

51 Jen, Y., Manova, K. \& Benezra, R. Each member of the Id gene family exhibits a unique expression pattern in mouse gastrulation and neurogenesis. Dev. Dyn. 208, 92-106 (1997).

52 Onda, H. et al. Tsc2 null murine neuroepithelial cells are a model for human tuber giant cells, and show activation of an mTOR pathway. Mol. Cell. Neurosci. 21, 561-574 (2002).

53 Uhlmann, E. J. et al. Heterozygosity for the tuberous sclerosis complex (TSC) gene products results in increased astrocyte numbers and decreased p27-Kip1 expression in TSC2+/- cells. Oncogene 21, 4050-4059 (2002).

54 Reynaud-Deonauth, S. et al. Notch signaling is involved in the regulation of $\mathrm{Id} 3$ gene transcription during Xenopus embryogenesis. Differentiation 69, 198-208 (2002).

55 Walker, A. S. et al. Nestin reporter transgene labels multiple central nervous system precursor cells. Neural Plasti. 2010, 894374 (2010).

56 Hockfield, S. \& McKay, R. D. Identification of major cell classes in the developing mammalian nervous system. J. Neurosci. 5, 3310-3328 (1985).

57 Dahiya, S., Lee da, Y. \& Gutmann, D. H. Comparative characterization of the human and mouse third ventricle germinal zones. J. Neuropathol. Exp. Neurol. 70, 622-633 (2011). 
58 Martynoga, B., Drechsel, D. \& Guillemot, F. Molecular control of neurogenesis: a view from the mammalian cerebral cortex. Cold Spring Harb. Perspect. Biol. 4, pii: a008359 (2012).

59 Howe, S. R. et al Rodent model of reproductive tract leiomyomata. Establishment and characterization of tumor-derived cell lines. Am. J. Pathol. 146, 1568-1579 (1995).

60 Yang, P. et al. Renal cell carcinoma in tuberous sclerosis complex. Am. J. Surg. Pathol. 38, 895-909 (2014).

61 Makovski, V., Haklai, R. \& Kloog, Y. Farnesylthiosalicylic acid (salirasib) inhibits Rheb in TSC2-null ELT3 cells: a potential treatment for lymphangioleiomyomatosis. Int. J. Cancer 130, 1420-1429 (2012).

62 Kwon, C. et al. Notch post-translationally regulates beta-catenin protein in stem and progenitor cells. Nat. Cell Biol. 13, 1244-1251 (2011).

63 Onda, H., Lueck, A., Marks, P. W., Warren, H. B. \& Kwiatkowski, D. J. Tsc2 $(+/-)$ mice develop tumors in multiple sites that express gelsolin and are influenced by genetic background. J. Clin. Invest. 104, 687-695 (1999).

64 Wilson, C. et al A mouse model of tuberous sclerosis 1 showing background specific early post-natal mortality and metastatic renal cell carcinoma. Hum. Mol. Genet. 14, 1839-1850 (2005).

65 Chan, S. M., Weng, A. P., Tibshirani, R., Aster, J. C. \& Utz, P. J. Notch signals positively regulate activity of the mTOR pathway in T-cell acute lymphoblastic leukemia. Blood 110, 278-286 (2007).

66 Patel, B. et al Exosomes mediate the acquisition of the disease phenotypes by cells with normal genome in tuberous sclerosis complex. Oncogene 35, 3027-3036 (2016).

67 Chen, J., Imanaka, N., Chen, J. \& Griffin, J. D. Hypoxia potentiates Notch signaling in breast cancer leading to decreased E-cadherin expression and increased cell migration and invasion. Br. J. Cancer 102, 351-360 (2010).

\section{Acknowledgements}

We acknowledge Aristotelis Astreinidis for comments, Deborah McClellan for editorial assistance, Maureen Murphy for providing H1299 cells, Navin Chintala, Sharad Sharma, and Kelly Hartley for technical assistance, the Developmental Corporation of Abilene for support, and the use of tissues procured by the NDRI supported by NIH 2 U42 OD011158. This work was supported by the National Institute of Health (R01CA190209 to M.M.M), the National Science Fundation (1052039 to B.W.), the Cancer Prevention and Research Institute of Texas (RP120168 to M.K.), the U.S. Department of Defense (TS140010 to M.K.), Laura W. Bush Institute for Women's Health (seed grants to M.M.M and M.K.).

\section{Author contributions}

Conception and design: J-H.C., E.P.H., F.R., M.M.M., B.W. and M.K. Development of methodology: J-H.C., B.P., S.B., S.M., M.P., S.G, F.R., K.G., D.J.K., B.W., M.M.M. and M. K. Acquisition of data: J-H.C., B.P., S.B., S.M., S.G., M.P., S.K.V., J.P., E.P.H., F.R., K.G., D.J.K., M.M.M., B.W. and M.K. Analysis and interpretation of data: J-H.C., B.P., S.B., S. M., Y.Z., M.P., S.G., E.P.H., F.R., K.G., D.J.K., H.M., M.M.M., B. W., and M.K. Writing and review of the manuscript: J-H.C., B.P., S.M., Y.Z., E.P.H., F.R., K.G., D.J.K., H.M., M. M.M., B.W. and M.K. Study supervision: M.M.M., B.W. and M.K.

\section{Additional information}

Supplementary Information accompanies this paper at https://doi.org/10.1038/s41467 017-01845-1.

Competing interests: The authors declare no competing financial interests.

Reprints and permission information is available online at http://npg.nature.com/ reprintsandpermissions/

Publisher's note: Springer Nature remains neutral with regard to jurisdictional claims in published maps and institutional affiliations.

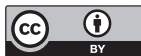

Open Access This article is licensed under a Creative Commons Attribution 4.0 International License, which permits use, sharing, adaptation, distribution and reproduction in any medium or format, as long as you give appropriate credit to the original author(s) and the source, provide a link to the Creative Commons license, and indicate if changes were made. The images or other third party material in this article are included in the article's Creative Commonslicense, unless indicated otherwise in a credit line to the material. If material is not included in the article'sCreative Commons license and your intended use is not permitted by statutory regulation or exceeds the permitted use, you will need to obtain permission directly from the copyright holder. To view a copy of this license, visit http://creativecommons.org/ licenses/by/4.0/.

(c) The Author(s) 2017 